\title{
Elliptic functions, area integrals and the exponential square class on $B_{1}(0) \subseteq \mathbb{R}^{n}, n>2$
}

\author{
by
}

\author{
Caroline Sweezy (Las Cruces, NM)
}

\begin{abstract}
For two strictly elliptic operators $L_{0}$ and $L_{1}$ on the unit ball in $\mathbb{R}^{n}$, whose coefficients have a difference function that satisfies a Carleson-type condition, it is shown that a pointwise comparison concerning Lusin area integrals is valid. This result is used to prove that if $L_{1} u_{1}=0$ in $B_{1}(0)$ and $S u_{1} \in L^{\infty}\left(S^{n-1}\right)$ then $\left.u_{1}\right|_{S^{n-1}}=f$ lies in the exponential square class whenever $L_{0}$ is an operator so that $L_{0} u_{0}=0$ and $S u_{0} \in L^{\infty}$ implies $\left.u_{0}\right|_{S^{n-1}}$ is in the exponential square class; here $S$ is the Lusin area integral. The exponential square theorem, first proved by Thomas Wolff for harmonic functions in the upper half-space, is proved on $B_{1}(0)$ for constant coefficient operator solutions, thus giving a family of operators for $L_{0}$. Methods of proof include martingales and stopping time arguments.
\end{abstract}

The subject treated here is the exponential square class for elliptic operator solutions in the unit ball in $\mathbb{R}^{n}$. The main result is given in Theorem 4 , where it is proved that if solutions for one operator $L_{0} u_{0}=0$ in $B_{1}(0)$ satisfy the exponential square result (i.e. if the Lusin area integral $S u_{0}$ is in $L^{\infty}\left(\partial B_{1}(0)\right)$ then $\left.u_{0}\right|_{\partial B_{1}(0)}=f$ lies in the exponential square class) then solutions $u_{1}$ to $L_{1} u_{1}=0$ in $B_{1}(0)$ will also satisfy the exponential square theorem if the coefficients of $L_{1}$ satisfy a Carleson-type condition with vanishing trace with respect to the coefficients of $L_{0}$. The main ingredient in the proof is to establish a comparison of area integrals by methods similar to those of Fefferman, Kenig and Pipher [FKP] in their proof of Dahlberg's preservation of $B^{q}$ for elliptic measures.

In order to ensure that the result is not vacuous, the exponential square theorem is proved on $B_{1}(0)$ for harmonic functions. The martingale argument of Chang, Wilson and Wolff [CWW] can be adapted to the geometry on $B_{1}(0)$. This result is established in part I in detail, using spherical coordinates. To establish the formula that served in place of the Calderón reproducing formula, and to establish the martingale proof, it seemed better to use spherical coordinates. A similar formula shows that solutions to constant coefficient equations also satisfy the exponential square result.

2000 Mathematics Subject Classification: 35J25, 42B25. 
Part II contains the proof of Theorem 4. This is done by using rectangular coordinates, since there is no particular need for this result to be proved in spherical coordinates, and the formulas in rectangular coordinates are much simpler. The cone formulas are somewhat different for the two coordinate systems, but are shown to compare with each other in the appendix. The Main Lemma used to prove Theorem 4 establishes a local pointwise comparison of area integrals for the two operator solutions, a result that may be of some independent interest. The other key estimate used in the proof of Theorem 4 is that if $F(x)$ is the difference of the two solutions, $F(x)=u_{1}(x)-u_{0}(x)$, then $\left(f_{R}|F(x)|^{2} d x\right)^{1 / 2} \leq C \varepsilon(\operatorname{diam} R) M_{\omega_{0}} S u_{1}\left(q_{0}\right)$ for any $\left.q_{0} \in \operatorname{proj} R\right|_{\partial B_{1}(0)}$, where $R$ is a Whitney-type region in $B_{1}(0)$ and $\varepsilon(\operatorname{diam} R)$ is the Carleson coefficient with an appropriate rate of decay as $\operatorname{diam} R \rightarrow 0$. This estimate can be used to prove a version of the good- $\lambda$ inequality in Lemma 2.16 of [FKP]. There is a similar proof for a version of Lemma 7 in [CS2].

I. Exponential square on the unit ball in $\mathbb{R}^{n}$. Suppose $u(y)$ is defined on $B_{1}(0) \subseteq \mathbb{R}^{n}$ so that

$$
\Delta u=0 \text { in } B_{1}(0),\left.\quad u\right|_{\partial B_{1}(0)}=f \in L^{1}\left(S^{n-1}\right),
$$

The Lusin area integral for $u$ is

where

$$
A_{\gamma} f\left(\theta_{x}\right)=A_{\gamma} u\left(\theta_{x}\right)=\left(\int_{\Gamma_{\gamma}^{\delta_{0}}\left(\theta_{x}\right)}\left|\nabla_{y} u(y)\right|^{2}\left(\operatorname{dist}\left(y, S^{n-1}\right)\right)^{2-n} d y\right)^{1 / 2},
$$

$$
\Gamma_{\gamma}^{\delta_{0}}\left(\theta_{x}\right)=\left\{(r, \theta): \delta_{0}<r<1,\left|\theta-\theta_{x}\right|<\gamma(1-r)\right\} .
$$

Then the following result is valid:

TheOREM 1. For $u$ and $f$ as above, suppose $A_{\gamma} f\left(\theta_{x}\right) \in L^{\infty}\left(S^{n-1}\right)$. Then there are constants $C_{1}, C_{2}>0$ independent of $Q$ and $f$ so that if $\ell(Q) \leq$ $\ell\left(Q_{0}\right)$ then

$$
\left(\frac{1}{|Q|} \int_{Q} \exp \left(\frac{C_{1}\left|f-f_{Q}\right|^{2}}{\left\|A_{\gamma} f\right\|_{\infty}^{2}}\right) d y\right)^{1 / 2}<C_{2}<\infty
$$

Here $Q$ is a surface cube on $S^{n-1}$ of limited size; it will be the image of a cube $\widehat{Q}$ in $\mathbb{R}^{n-1}$ under the projection map $\widehat{P}$ defined below.

Theorem 1 can be proved by using a martingale result similar to the one in $[\mathrm{CWW}]$ along with other estimates as in $[\mathrm{BM}]$. In fact, one can obtain a version of the Calderón reproducing formula so that

$$
\int_{r=1-\varepsilon} u\left(r \theta_{y}\right) P_{1-r}\left(\theta_{x}-\theta_{y}\right) d \theta_{y}
$$

equals a sum of eight integrals - three boundary integrals and five integrals over a solid spherical shell region $R$ in $B_{1}(0)$. (See Lemma 1 below; $P_{1-r}$ is 
defined after the statement of Theorem 2.) Then $P_{1-r}(\theta)$ being an approximation to the identity implies that, as $r \rightarrow 1, \int_{r=1-\varepsilon} u\left(r \theta_{y}\right) P_{1-r}\left(\theta_{x}-\theta_{y}\right) d \theta_{y}$ $\rightarrow f\left(\theta_{x}\right)$. This happens because $u$ converges non-tangentially to the boundary function $f$.

So it suffices to prove that $\int_{r=1-\varepsilon} u\left(r \theta_{y}\right) P_{1-r}\left(\theta_{x}-\theta_{y}\right) d \theta_{y}$ is in the exponential square class. This can be shown by the following steps. First all three boundary integrals $I_{i}, i=1,2,3$, in Lemma 1 can be dominated by the area integral, i.e. $\left|I_{i}-I_{i, Q}\right| \leq c\left\|A_{\gamma} f\right\|_{\infty}$ a.e. Next two of the integrals over $R$ can be bounded directly by $c A_{\gamma} f\left(\theta_{x}\right)$ also. The remaining three integrals over $R$ can be replaced by "dyadic" martingales with an error of $c\left\|A_{\gamma} f\right\|_{\infty}$ a.e. These martingales have their dyadic square functions bounded by $c\left\|A_{\gamma} f\right\|_{\infty}$ for a.e. $\theta_{x}$, and so Theorem 2 below shows that the martingales are exponentially square integrable.

Note that each function bounded by $c\left\|A_{\gamma} f\right\|_{\infty}$ is in the exponential square class and any finite sum of exponentially square integrable functions is also exponentially square integrable; this implies that $\int_{r=1-\varepsilon} u P_{1-r}$ is in the exponential square class. The constants will be shown to be independent of $\varepsilon$, so $f\left(\theta_{x}\right)$ is in the exponential square class also.

The steps of this proof are well known by now ([CWW], $[\mathrm{BM}])$. The intention in this paper is to make clear the elements of the proof that change for $B_{1}(0) \subseteq \mathbb{R}^{n}, n \geq 3$, and to show that the standard proof works in this slightly new setting.

To begin, a version of the dyadic martingale exponential square result must be established. To create $\sigma$-algebras $\mathcal{F}_{m}$ on $S^{n-1}$, with $\mathcal{F}_{m-1} \subseteq \mathcal{F}_{m}$, let $S_{0}$ be a fixed polar cap on the lower hemisphere of $S^{n-1}$. The cap must be large enough to contain $3 Q$ for any surface cube $Q$, where $3 Q$ is the image of $3 \widehat{Q}$ under the projection operator $\widehat{P}$ defined by

$$
\widehat{P}(x)=\widehat{P}\left(x_{1}, \ldots, x_{n-1},-1\right)=\left(x_{1}, x_{2}, \ldots, x_{n-1},-\left(1-\sum_{i=1}^{n-1} x_{i}^{2}\right)^{1 / 2}\right)
$$

so $\widehat{P}$ maps $\mathbb{R}^{n-1} \times\{-1\}$ onto the lower hemisphere of $S^{n-1}$.

Now $\widehat{Q}$ will denote a dyadic subcube of an initial cube $3 \widehat{Q}_{0}$, where $\widehat{Q}_{0}$ is a cube of fixed size centered at $(0, \ldots, 0,-1)$ in $\mathbb{R}^{n-1} \times\{-1\}$, and $3 \widehat{Q}_{0}$ is the cube concentric with $\widehat{Q}_{0}$ and with side length $\ell\left(3 \widehat{Q}_{0}\right)=3 \ell\left(\widehat{Q}_{0}\right)$.

To define the $\sigma$-algebras, let $\mathcal{F}_{m}$ be the $\sigma$-algebra generated by the collection $\widehat{P}\left(\widehat{Q}_{m}\right)$, where $\widehat{Q}_{m}$ is a dyadic subcube of $3 \widehat{Q}_{0} \subseteq \mathbb{R}^{n-1} \times\{-1\}$ of side length $\ell\left(\widehat{Q}_{m}\right)=2^{-m} \ell\left(\widehat{Q}_{0}\right)$. The projection function $\widehat{P}$ is $1-1$ on $3 \widehat{Q}_{0}$ and maps $\cup \widehat{Q}_{m}$ onto $\widehat{P}\left(3 \widehat{Q}_{0}\right)$ in $S_{0}$, for each $m$. In this way the $\mathcal{F}_{m}$ are simply the usual dyadic $\sigma$-algebras in $\mathbb{R}^{n-1}$ mapped to $S_{0}$ by $\widehat{P}$. The distortion produced by $\widehat{P}$ in $S_{0}$ is bounded, so that $\ell(P(\widehat{Q}))=\ell(Q) \sim \ell(\widehat{Q})$ and 
$|Q| \sim|\widehat{Q}|$. Here $\ell(Q)$ can be taken to be the diameter of $Q$, and $|Q|$ denotes the Lebesgue surface measure of $Q$.

It is easy to see that $\mathcal{F}_{m-1} \subseteq \mathcal{F}_{m}$ for all $m \geq 1$. Given the sequence $\left\{\mathcal{F}_{m}\right\}_{m=0}^{\infty}$ of $\sigma$-algebras, martingales are defined in the usual way, being a sequence $\left\{f_{m}\right\}_{m=0}^{\infty}$ of functions defined on $S_{0}$, so that each $f_{m}$ is $\mathcal{F}_{m^{-}}$ measurable and $E\left(f_{m+1} \mid \mathcal{F}_{m}\right)=f_{m}, m=0,1,2, \ldots$ If $\lim _{m \rightarrow \infty} f_{m}\left(\theta_{x}\right)$ $=f\left(\theta_{x}\right)$ a.e. $\theta_{x}$, then $f$ is the martingale limit function. Also any $L^{1}$ function $f\left(\theta_{x}\right)$ can be taken to generate a martingale. By a mild abuse of terminology the term dyadic will continue to be used here; this dyadic martingale is

$$
f_{m}=\sum_{Q_{m}^{j} \text { are generators of } \mathcal{F}_{m}} \frac{1}{\left|Q_{m}^{j}\right|} \int_{Q_{m}^{j}} f(\theta) d \theta \cdot \chi_{Q_{m}^{j}},
$$

where $\left|Q_{m}^{j}\right|=\int_{Q_{m}^{j}} d \theta_{y}$. The $\left\{f_{m}\right\}_{m=0}^{\infty}$ have $f\left(\theta_{x}\right)$ as their martingale limit function.

The dyadic martingale square function for any dyadic martingale $\left\{g_{m}\right\}$ is defined by

$$
S_{m}=S g_{m}(\theta)=\left(\sum_{j=1}^{m}\left\|d_{j} \chi_{Q_{j-1}}\right\|_{\infty}^{2}\right)^{1 / 2}, \quad d_{j}=g_{j}-g_{j-1} .
$$

If $g_{m}$ has a martingale limit function $g$ then

$$
S g(\theta)=\left(\sum_{j=1}^{\infty}\left\|d_{j} \chi_{Q_{j-1}}\right\|_{\infty}^{2}\right)^{1 / 2} .
$$

But $S g(\theta)$ is well defined even if $g$ is not known.

THEOREM 2. If $\left\{f_{m}\right\}_{m=0}^{\infty}$ is a dyadic martingale as defined above on $Q \subseteq S_{0} \subseteq S^{n-1}$ with limit function $f\left(\theta_{x}\right)$, suppose $S f \in L^{\infty}(Q)$. Then there are constants $C_{1}, C_{2}>0$ independent of $Q$ and $f$ so that

$$
\frac{1}{|Q|} \int_{Q} \exp \left\{\frac{C_{1}\left|f\left(\theta_{x}\right)-f_{Q}\right|^{2}}{\|S f\|_{\infty}^{2}}\right\} d \theta_{x}<C_{2}<\infty .
$$

The proof is the same argument used in $[\mathrm{CWW}]$ and $[\mathrm{BM}]$ to establish this result for dyadic martingales in $\mathbb{R}^{n}$.

To establish the result of Theorem 1 using Theorem 2, an essential ingredient is to find a form of the Calderón reproducing formula. The following calculation uses the Laplacian on a sphere; the spherical form of the Laplacian is

$$
\begin{aligned}
\Delta= & \frac{\partial^{2}}{\partial r^{2}}-\frac{1}{r^{2}} \sum \sum \theta_{j} \theta_{k} \frac{\partial^{2}}{\partial \theta_{j} \partial \theta_{k}}+\frac{n-1}{r} \frac{\partial}{\partial r} \\
& +\frac{1}{r^{2}} \sum \frac{\partial^{2}}{\partial \theta_{j}^{2}}-\frac{n-1}{r^{2}} \sum \theta_{k} \frac{\partial}{\partial \theta_{k}} .
\end{aligned}
$$


The sums are all taken from 1 to $n-1$ for each index. Here $y_{j}=r \theta_{j}$ for $j=1, \ldots, n, \theta_{j}=\cos \psi_{j}$ for $j=1, \ldots, n-1$ and $\theta_{n}=\left(1-\sum_{j=1}^{n-1} \theta_{j}^{2}\right)^{1 / 2}$. The angles $\psi_{j}$ are the angles with respect to each coordinate axis. (See Helm's book $[\mathrm{H}]$.)

To produce a substitute for the Calderón reproducing formula, following the method in Bañuelos and Moore [BM], take $k(\theta)$ defined on $S^{n-1}$ to be smooth, radial, of compact support, with $\int k(\theta) d \theta=1$, and let

$$
k_{1-r}(\theta)=\frac{1}{(1-r)^{n-1}} k\left(\frac{\theta}{1-r}\right) \text {. }
$$

Then define

$$
P_{1-r}(\theta)=\frac{\partial}{\partial r}\left\{\frac{1}{(1-r)^{n-2}} k\left(\frac{\theta}{1-r}\right)\right\}=\frac{1}{(1-r)^{n-1}} P\left(\frac{\theta}{1-r}\right) .
$$

This gives

$$
P(\theta)=(n-2) k(\theta)+\sum \theta_{k} \frac{\partial}{\partial \theta_{k}} k(\theta)
$$

as long as $\left(\theta_{k} \frac{\partial}{\partial \theta_{k}} k(\theta)\right)_{1-r}$ is understood to be

$$
\frac{1}{(1-r)^{n-1}} \frac{\theta_{j}}{1-r} \frac{\partial k(\theta /(1-r))}{\partial\left(\theta_{j} /(1-r)\right)} \text {. }
$$

Then

$$
\begin{aligned}
\frac{\partial P_{1-r}}{\partial r}(\widehat{\theta})= & \frac{\partial}{\partial r}\left\{\frac{1}{(1-r)^{n-1}} P\left(\frac{\theta_{x}-\theta_{y}}{1-r}\right)\right\}=\frac{n-1}{(1-r)^{n}} P\left(\frac{\theta_{x}-\theta_{y}}{1-r}\right) \\
& +\frac{1}{(1-r)^{n-1}} \sum \frac{\partial}{\partial r}\left(\frac{\theta_{x, j}-\theta_{y, j}}{1-r}\right) \frac{\partial P(\widehat{\theta})}{\partial \widehat{\theta}_{j}} \\
= & \frac{n-1}{(1-r)^{n}} P(\widehat{\theta})+\frac{1}{(1-r)^{n}} \sum \widehat{\theta}_{j} \frac{\partial P(\widehat{\theta})}{\partial \widehat{\theta}_{j}} \\
= & \frac{1}{(1-r)^{n}} \sum \frac{\partial}{\partial \widehat{\theta}_{j}}\left(\widehat{\theta}_{j} P(\widehat{\theta})\right),
\end{aligned}
$$

where

$$
\widehat{\theta}_{j}=\frac{\theta_{x, j}-\theta_{y, j}}{1-r}, \quad \widehat{\theta}=\frac{\theta_{x}-\theta_{y}}{1-r} .
$$

Notice that

$$
\int k_{1-r}\left(\theta_{x}-\theta_{y}\right) d \theta_{x}=1, \quad \int P_{1-r}\left(\theta_{x}-\theta_{y}\right) d \theta_{x}=1
$$

and $\int \frac{\partial}{\partial r} P_{1-r} d \theta_{x}=0$. 
Integrating from $r=1-\varepsilon$ to $r=\delta_{0}$ gives

$$
\begin{aligned}
\int_{r=\delta_{0}} u P_{1-r}-\int_{r=1-\varepsilon} u P_{1-r} & =\int_{1-\varepsilon}^{\delta_{0}} \frac{\partial}{\partial r} \int_{\operatorname{supp} P_{1-r}} u(r \theta) P_{1-r}(\widehat{\theta}) d \theta d r \\
= & \int_{1-\varepsilon}^{\delta_{0}} \int_{\operatorname{supp} P_{1-r}}\left\{\frac{\partial u}{\partial r} P_{1-r}+u \frac{\partial P_{1-r}}{\partial r}\right\} d \theta d r \\
= & \iint_{R} \frac{\partial u}{\partial r} \frac{\partial}{\partial r}\left(\frac{1}{(1-r)^{n-2}} k(\widehat{\theta})\right) d \theta d r \\
& +\iint_{R} u \frac{1}{(1-r)^{n}} \sum \frac{\partial}{\partial \widehat{\theta}_{j}}\left(\widehat{\theta}_{j} P(\widehat{\theta})\right) d \theta d r=I+I I,
\end{aligned}
$$

where

$$
R=\left\{(r, \theta): 1-\varepsilon>r>\delta_{0}, \theta_{x} \in \beta Q_{0}\right\},
$$

and $\beta>1$ is chosen so that $\operatorname{supp} k_{1-r}\left(\theta_{x}-*\right)$ lies inside

$$
\left\{(r, \theta): \delta<r<1, \theta \in \beta Q_{0}\right\} \quad \text { if } \theta_{x} \in Q_{0} .
$$

Here $\delta$ is a fixed radius, but $\delta_{0}$ depends on the cube $Q$ under consideration. For all cubes $Q, \delta \leq \delta_{0} / 2$. For the purpose of Lemma 1 below, consider $Q_{0}$ to be fixed.

Next, to produce integrals in forms that can be dealt with by the martingale replacement method (as in $[\mathrm{BM}]$ ), or that can be estimated directly, integration by parts is used on both terms. The first integral becomes

$$
\begin{aligned}
I= & \int_{r=\delta_{0}}\left(\frac{\partial}{\partial r} u\right)\left(\frac{1}{(1-r)^{n-2}} k(\widehat{\theta})\right) d \theta \\
& -\int_{r=1-\varepsilon}\left(\frac{\partial}{\partial r} u\right)\left(\frac{1}{(1-r)^{n-2}} k(\widehat{\theta})\right) d \theta-\iint_{R}\left(\frac{\partial^{2}}{\partial r^{2}} u(r \theta)\right) \frac{1}{(1-r)^{n-2}} k(\theta) d \theta d r \\
= & \int_{r=\delta_{0}}\left(\frac{\partial}{\partial r} u\right)\left(\frac{1}{(1-r)^{n-2}} k(\widehat{\theta})\right) d \theta-\int_{r=1-\varepsilon}\left(\frac{\partial}{\partial r} u\right)\left(\frac{1}{(1-r)^{n-2}} k(\widehat{\theta})\right) d \theta \\
& +(n-1) \iint_{R} \frac{1}{(1-r)^{n-2}} \frac{1}{r}\left(\frac{\partial}{\partial r} u\right) k(\widehat{\theta}) d \theta d r \\
& +\iint_{R} \frac{1}{(1-r)^{n-2}} k(\widehat{\theta}) \frac{1}{r^{2}} \sum \frac{\partial^{2}}{\partial \theta_{j}^{2}} u(r \theta) d \theta d r \\
& -\iint_{R} \frac{1}{(1-r)^{n-2}} k(\widehat{\theta}) \frac{1}{r^{2}} \sum \sum \theta_{j} \theta_{k} \frac{\partial^{2}}{\partial \theta_{j} \partial \theta_{k}} u(r \theta) d \theta d r \\
& -(n-1) \iint_{R} \frac{1}{(1-r)^{n-2}} k(\widehat{\theta}) \frac{1}{r^{2}} \sum \theta_{k} \frac{\partial}{\partial \theta_{k}} u(r \theta) .
\end{aligned}
$$


The expression substituted for $-\frac{\partial^{2}}{\partial r^{2}} u(r \theta)$ has been taken from the Laplacian as given above.

Now using integration by parts again on the two integrals in the last expression that involve second derivatives in $u$ gives

$$
\begin{aligned}
& I=\int_{r=\delta_{0}}\left(\frac{\partial}{\partial r} u\right)\left(\frac{1}{(1-r)^{n-2}} k(\widehat{\theta})\right) d \theta-\int_{r=1-\varepsilon}\left(\frac{\partial}{\partial r} u\right)\left(\frac{1}{(1-r)^{n-2}} k(\widehat{\theta})\right) d \theta \\
& +(n-1) \iint_{R} \frac{1}{(1-r)^{n-2}} \frac{1}{r}\left(\frac{\partial}{\partial r} u\right) k(\widehat{\theta}) d \theta d r \\
& -\iint_{R} \frac{1}{(1-r)^{n-2}} \frac{1}{r^{2}}\left(\sum \frac{\partial}{\partial \theta_{j}} k\left(\frac{\theta_{x, j}-\theta_{y, j}}{1-r}\right)\right) \frac{\partial}{\partial \theta_{j}} u(r \theta) d \theta d r \\
& +n \iint_{R} \frac{1}{(1-r)^{n-2}} \frac{1}{r^{2}} k(\widehat{\theta}) \sum \theta_{k} \frac{\partial}{\partial \theta_{k}} u(r \theta) d \theta d r \\
& +\iint_{R} \frac{1}{(1-r)^{n-2}} \frac{1}{r^{2}} \sum \sum\left(\frac{\partial}{\partial \theta_{j}} k\left(\frac{\theta_{x, j}-\theta_{y, j}}{1-r}\right)\right) \theta_{j} \theta_{k} \frac{\partial}{\partial \theta_{k}} u(r \theta) d \theta d r \\
& -(n-1) \iint_{R} \frac{1}{(1-r)^{n-2}} \frac{1}{r^{2}} k(\widehat{\theta}) \sum \theta_{k} \frac{\partial}{\partial \theta_{k}} u(r \theta) d \theta d r \\
& =\int_{r=\delta_{0}}\left(\frac{\partial}{\partial r} u\right)\left(\frac{1}{(1-r)^{n-2}} k(\widehat{\theta})\right) d \theta-\int_{r=1-\varepsilon}\left(\frac{\partial}{\partial r} u\right)\left(\frac{1}{(1-r)^{n-2}} k(\widehat{\theta})\right) d \theta \\
& -\iint_{R} \frac{1}{(1-r)^{n-2}} \frac{1}{r^{2}} \sum\left(\frac{\partial}{\partial \theta_{j}} k\left(\frac{\theta_{x, j}-\theta_{y, j}}{1-r}\right)\right) \frac{\partial}{\partial \theta_{j}} u(r \theta) d \theta d r \\
& +\iint_{R} \frac{1}{(1-r)^{n-2}} \frac{1}{r^{2}} \sum \sum\left(\frac{\partial}{\partial \theta_{j}} k\left(\frac{\theta_{x, j}-\theta_{y, j}}{1-r}\right)\right) \theta_{j} \theta_{k} \frac{\partial}{\partial \theta_{k}} u(r \theta) d \theta d r \\
& +(n-1) \iint_{R} \frac{1}{(1-r)^{n-2}} \frac{1}{r}\left(\frac{\partial}{\partial r} u\right) k(\widehat{\theta}) d \theta d r \\
& +\iint_{R} \frac{1}{(1-r)^{2}} \frac{1}{r^{2}} k(\widehat{\theta})\left(\sum \theta_{k} \frac{\partial}{\partial \theta_{k}} u(r \theta)\right) d \theta d r
\end{aligned}
$$

All sums are from 1 to $n-1, \theta=\theta_{y}$ and $\theta_{j}=\theta_{y, j}$.

The boundary terms can be dominated by the Lusin area integral for $u$ and the first two integrals over $R$ in the last expression can be estimated by using the method of replacing them by a martingale with an error bounded by a constant times the Lusin area integral of $u$. The essential properties for doing this are that the kernels $\frac{\partial}{\partial \widehat{\theta}_{j}} k(\widehat{\theta})$ have $\int \frac{\partial}{\partial \widehat{\theta}_{j}} k(\widehat{\theta}) d \theta_{x}=0$ and that the dilation factors, $1 /(1-r)^{n-2}$, are the right magnitude for domination by the area integral. 
However, the last integral can be bounded directly by $A_{\gamma} u\left(\theta_{x}\right)$. Breaking $R=\left\{(r, \theta): 1-\varepsilon>r>\delta_{0}, \theta \in \beta Q_{0}\right\}$ into dyadic approach regions $T_{Q}$ (annular segments of approximately the same geometric shape as $R$, whose dimension compares with their distance from $S^{n-1}$ ), one easily sees that

$$
\begin{aligned}
& \left|\iint_{R} \frac{1}{(1-r)^{n-2}} \frac{1}{r} k(\widehat{\theta}) \frac{\partial u}{\partial r} d \theta d r\right| \\
& =\left|\sum_{Q \subset S^{n-1}} \iint_{T_{Q} \cap \operatorname{supp} k(\widehat{\theta})} \frac{1}{(1-r)^{n-2}} \frac{1}{r} k(\widehat{\theta}) \frac{\partial u}{\partial r} d \theta d r\right| \\
& \leq \sum_{Q \subset S^{n-1}}\left(\int_{T_{Q} \cap \operatorname{supp} k(\widehat{\theta})}\left|\frac{\partial u}{\partial r}\right|^{2}(1-r)^{2-n} d \theta d r\right)^{1 / 2} \\
& \quad \times\left(\iint_{T_{Q} \cap \operatorname{supp} k(\widehat{\theta})} \frac{1}{r^{2}} \frac{1}{(1-r)^{n-2}}\left(k\left(\frac{\theta_{x}-\theta_{y}}{1-r}\right)\right)^{2} d \theta d r\right)^{1 / 2} .
\end{aligned}
$$

In $T_{Q}$ we have $\left(k\left(\frac{\theta_{x}-\theta_{y}}{1-r}\right)\right)^{2} \leq c$, and $1 / r^{2} \leq 1 / \delta_{0}^{2}$, so this implies that

$$
\begin{aligned}
& \left(\underset{T_{Q} \cap \operatorname{supp} k(\widehat{\theta})}{\int r^{2}} \frac{1}{(1-r)^{n-2}}\left(k\left(\frac{\theta_{x}-\theta_{y}}{1-r}\right)\right)^{2} d \theta d r\right)^{1 / 2} \\
& \quad \geq c\left\{\frac{\left|T_{Q}\right|}{\delta_{0}^{2} \inf (1-r)^{n-2}}\right\}^{1 / 2} \leq c^{\prime} \ell(Q)
\end{aligned}
$$

since $\left|T_{Q}\right|$ compares with $(1-r)^{n}$ if $r \theta \in T_{Q}$. The $\inf (1-r)^{n-2}$ is also taken over $r \theta \in T_{Q}$. So

$$
\begin{aligned}
& \left|\iint_{R} \frac{1}{(1-r)^{n-2}} \frac{1}{r} k(\widehat{\theta}) \frac{\partial u}{\partial r} d \theta d r\right| \\
& \quad \leq c^{\prime} \sum_{Q \subset S^{n-1}}\left(\iint_{T_{Q} \cap \operatorname{supp} k(\widehat{\theta})}\left|\frac{\partial u}{\partial r}\right|^{2}(1-r)^{2-n} d \theta d r\right)^{1 / 2} \ell(Q) \\
& \quad \leq c^{\prime \prime}\left(\sum_{Q \subset S^{n-1}} \iint_{T_{Q} \cap \operatorname{supp} k(\widehat{\theta})}\left|\frac{\partial u}{\partial r}\right|^{2}(1-r)^{2-n} d \theta d r\right)^{1 / 2}\left(\sum_{T_{Q} \cap \Gamma_{\gamma}^{\delta_{0}}\left(\theta_{x}\right) \neq \emptyset} \ell(Q)^{2}\right)^{1 / 2} \\
& \quad \leq c\left(\delta_{0}\right)\left(\int_{\Gamma_{\gamma}^{\delta}\left(\theta_{x}\right)}|\nabla u|^{2}(1-r)^{2-n} d \theta d r\right)^{1 / 2} \sim A_{\gamma} u\left(\theta_{x}\right)
\end{aligned}
$$

as long as $\theta_{x} \in Q_{0}$ since $\beta Q_{0}$ stays well inside the lower half of $S^{n-1}$ (see the Appendix). 
For the integral $I I$, integration by parts gives

$$
\begin{aligned}
I I & =-\iint_{R} \frac{1}{(r-1)^{n}} \sum\left(\frac{\partial}{\partial \widehat{\theta}_{j}} u(r \theta)\right) \widehat{\theta}_{j} P(\widehat{\theta}) \\
& =\iint_{R} \frac{1}{(r-1)^{n-1}} \sum\left(\frac{\partial}{\partial \theta_{j}} u(r \theta)\right) \widehat{\theta}_{j} P(\widehat{\theta}),
\end{aligned}
$$

where $\widehat{\theta}_{j}$ and $\widehat{\theta}$ are as before. The latter integral should be easy to estimate using the martingale method, described above. $\widehat{\theta}_{j} P(\widehat{\theta})$ is odd in the $\theta_{j}$ variable so that $\int \widehat{\theta}_{j} P(\widehat{\theta}) d \theta_{x}=0$. Also in this integrand $1 /(1-r)^{n-1}$ is the correct dilation for later estimations by Lipschitz norms.

The Lusin area integral equals

$$
\begin{aligned}
A_{\gamma} u\left(\theta_{x}\right)= & \left(\int_{\Gamma_{\gamma}^{\delta}\left(\theta_{x}\right)}\left|\nabla_{y} u\right|^{2}\left(\operatorname{dist}\left(y, S^{n-1}\right)\right)^{2-n} d y\right)^{1 / 2} \\
=\left(\int_{\Gamma_{\gamma}^{\delta}\left(\theta_{x}\right)}\left\{\left(\frac{\partial}{\partial r} u\right)^{2}+\frac{1}{r^{2}} \sum\left(\frac{\partial}{\partial \theta_{j}} u\right)^{2}-\frac{1}{r^{2}}\left(\sum \theta_{j} \frac{\partial}{\partial \theta_{j}} u\right)^{2}\right\}\right. & \left.\times \frac{r^{n-1}}{(1-r)^{n-2}} d r d \sigma(\theta)\right)^{1 / 2}
\end{aligned}
$$

in spherical coordinates, where

$$
\Gamma_{\gamma}^{\delta}\left(\theta_{x}\right)=\left\{(r, \theta): \delta<r<1,\left|\theta-\theta_{x}\right|<\gamma(1-r)\right\}
$$

$\Gamma_{\gamma}^{\delta}\left(\theta_{x}\right)$ is not a Euclidean cone, but it can be compared with Euclidean cones of different apertures as long as $\delta>0$ is fixed. Here, as above, $\delta \leq \delta_{0} / 2$. Moreover, $A_{\gamma} u\left(\theta_{x}\right)$ can be shown to compare to

$$
\left(\int_{\Gamma_{\gamma}^{\delta}\left(\theta_{x}\right)}\left\{\left(\frac{\partial}{\partial r} u\right)^{2}+\frac{1}{r^{2}} \sum\left(\frac{\partial}{\partial \theta_{j}} u\right)^{2}\right\} \frac{r^{n-1}}{(1-r)^{n-2}} d r d \theta\right)^{1 / 2}
$$

by using the elementary estimate

$$
\left(\sum \theta_{j} \frac{\partial}{\partial \theta_{j}} u\right)^{2} \leq \alpha(n) \sum\left(\frac{\partial}{\partial \theta_{k}} u\right)^{2}, \quad \text { where } \quad \alpha(n)=1-\theta_{n}^{2}<1
$$

because the region on $S^{n-1}$ under consideration will always be well inside the lower hemisphere. Also $d \sigma(\theta)=d \theta /\left|\theta_{n}\right| \sim d \theta$ in the region under consideration.

Altogether the formula for approximating the boundary function $f\left(\theta_{x}\right)$, using the non-tangential convergence of $u(r \theta)$ to $f\left(\theta_{x}\right)$, is given in Lemma 1: 
LEMMA 1.

$$
\begin{aligned}
& \int_{r=1-\varepsilon} u\left(r \theta_{y}\right) P_{1-r}\left(\theta_{x}-\theta_{y}\right) d \theta_{y}=\int_{r=\delta_{0}} u\left(r \theta_{y}\right) P_{1-r}\left(\theta_{x}-\theta_{y}\right) d \theta_{y} \\
& -\int_{r=\delta_{0}}\left(\frac{\partial}{\partial r} u\right)\left(\frac{1}{(1-r)^{n-2}} k(\widehat{\theta})\right) d \theta-\int_{r=1-\varepsilon}\left(\frac{\partial}{\partial r} u\right)\left(\frac{1}{(1-r)^{n-2}} k(\widehat{\theta})\right) d \theta \\
& -\iint_{R} \frac{1}{(1-r)^{n-2}} \frac{1}{r^{2}} \sum\left(\frac{\partial}{\partial \theta_{j}} k\left(\frac{\theta_{x}-\theta_{y}}{1-r}\right)\right) \frac{\partial}{\partial \theta_{j}} u(r \theta) d \theta d r \\
& +\int_{R} \frac{1}{(1-r)^{n-2}} \frac{1}{r^{2}} \sum \sum\left(\frac{\partial}{\partial \theta_{j}} k\left(\frac{\theta_{x}-\theta_{y}}{1-r}\right)\right) \theta_{j} \theta_{k} \frac{\partial}{\partial \theta_{k}} u(r \theta) d \theta d r \\
& +(n-1) \iint_{R} \frac{1}{(1-r)^{n-2}} \frac{1}{r}\left(\frac{\partial}{\partial r} u\right) k(\widehat{\theta}) d \theta d r \\
& +\iint_{R} \frac{1}{(1-r)^{n-1}} \sum\left(\frac{\partial u}{\partial \theta_{j}}\right) \widehat{\theta}_{j} P(\widehat{\theta}) d \theta d r .
\end{aligned}
$$

The last integral over $R$ has been shown to be dominated by $c A_{\gamma} f\left(\theta_{x}\right)$. The three boundary integrals will be proved to have the same upper bound. The remaining two integrals over $R$ can be shown to differ from dyadic martingales by an error that is bounded by $C A_{\gamma} f\left(\theta_{x}\right)$; the dyadic martingales have square functions that are bounded by $C A_{\gamma} f\left(\theta_{x}\right)$. The constants $C$ are independent of $\varepsilon$ and $\theta_{x}$. The method that will be used to prove these facts is essentially the one in $[\mathrm{CWW}]$ and $[\mathrm{BM}]$, with some relatively minor changes due to the geometry of $B_{1}(0)$.

To estimate the boundary terms in the formula of Lemma 1, first $\int_{r=\delta_{0}} u P_{1-r}-\left(\int_{r=\delta_{0}} u P_{1-r}\right)_{Q}$ is bounded by $c\left\|A_{\gamma} f\right\|_{\infty}$ because

$$
\begin{aligned}
& \mid \int_{r=\delta_{0}} u\left(r \theta_{y}\right) \frac{1}{(1-r)^{n-1}} P\left(\frac{\theta_{x}-\theta_{y}}{1-r}\right) d \theta_{y} \\
& \quad-\int_{Q} \int_{r=\delta_{0}} u\left(r \theta_{y}\right) P_{1-r}\left(\theta_{z}-\theta_{w}\right) d \theta_{w} d \theta_{z} \mid \\
& \leq \frac{1}{|Q|} \int_{Q}\left|\int_{r=\delta_{0}} u\left(r \theta_{y}\right) P\left(\frac{\theta_{x}-\theta_{y}}{1-r}\right) d \theta_{y}-\int_{r=\delta_{0}} u\left(r \theta_{w}\right) P_{1-r}\left(\theta_{z}-\theta_{w}\right) d \theta_{w}\right| d \theta_{z} \\
& =\left(\operatorname{letting} \underline{\theta}_{x}=\theta_{x}-\theta_{y} \text { and } \underline{\theta}_{z}=\theta_{z}-\theta_{w}\right) \\
& \quad \frac{1}{|Q|} \int_{Q}-\int_{r=\delta_{0}} u\left(r\left(\theta_{x}-\underline{\theta}_{x}\right)\right) P_{1-r}\left(\underline{\theta}_{x}\right) d \underline{\theta}_{x} \\
& \quad+\int_{r=\delta_{0}} u\left(r\left(\theta_{z}-\underline{\theta}_{z}\right)\right) \cdot P_{1-r}\left(\underline{\theta}_{z}\right) d \underline{\theta}_{z} \mid d \theta_{z}
\end{aligned}
$$




$$
\begin{aligned}
& \leq \frac{1}{|Q|} \int_{Q} \int_{r=\delta_{0}} P_{1-r}\left(\underline{\theta}_{z}\right)\left|u\left(r\left(\theta_{x}-\underline{\theta}_{x}\right)\right)-u\left(r\left(\theta_{z}-\underline{\theta}_{z}\right)\right)\right| d \underline{\theta}_{z} d \theta_{z} \\
& \leq \frac{1}{|Q|} \int_{Q} \int_{r=\delta_{0}} \frac{1}{(1-r)^{n-1}} P\left(\frac{\underline{\theta}_{z}}{1-r}\right)\left|\nabla_{\theta} u\left(\theta^{*}\right)\right|\left|\theta_{x}-\theta_{z}+\underline{\theta}_{z}-\underline{\theta}_{x}\right| \\
& \leq \sup _{\theta^{*} \in Q}\left|\nabla_{\theta} u\left(\theta^{*}\right)\right| \cdot c \ell(Q) \leq C\left\|A_{\gamma} f\right\|_{\infty} .
\end{aligned}
$$

The last inequality follows from an estimate for harmonic functions that appears in Stein [S]. The result is stated here (Lemma 2) for derivatives in spherical coordinates.

The second to the last inequality is valid since supp $P\left(\theta_{x}-\theta_{y}\right) \subseteq 3 Q$ when $\theta_{x} \in Q$ and also $\left|\theta_{x}-\underline{\theta}_{x}-\left(\theta_{z}-\underline{\theta}_{z}\right)\right|=\left|\theta_{y}-\theta_{w}\right| \leq c \ell(Q) \leq c\left(1-\delta_{0}\right)$ by definition of $\delta_{0}$, taking $\delta_{0}=1-\left(\frac{r_{0}+12 \sqrt{n-1}}{r_{0} \gamma}\right) \ell(Q)$ as below. Here $r_{0}$ is a bound for the distortion produced by $\widehat{P}$.

LEMMA 2 (Stein). If $u$ is harmonic in $\Gamma_{\gamma}^{\delta}\left(\theta_{x}\right)$ then for any point $r \theta_{y} \in$ $\Gamma_{\alpha \gamma}^{\delta / 2}\left(\theta_{x}\right)$ both

$$
|1-r|\left|\frac{\partial u}{\partial r}\right|,|1-r|\left|\nabla_{\theta} u\right| \leq c A_{\gamma} f\left(\theta_{x}\right), \quad c=c(\alpha) .
$$

Proof. Use the mean value property of the harmonic functions $\partial u / \partial r$ and $\partial u / \partial \theta_{j}, j=1, \ldots, n-1$, and Cauchy-Schwarz.

Lemma 2 and the fact that $\int k_{1-r}(\theta) d \theta=1$ give an upper bound of $c\left\|A_{\gamma} f\right\|_{\infty}$ for both boundary integrals $\int_{r=\varrho}(1-r) \frac{\partial u}{\partial r} k_{1-r}\left(\theta_{x}-\cdot\right), \varrho=\delta_{0}$ and $1-\varepsilon$.

The three integrals

$$
\iint_{R} \frac{1}{r^{2}(1-r)^{n-2}} \sum_{j=1}^{n-1} \frac{\partial u}{\partial \theta_{j}} \frac{\partial k}{\partial \theta_{j}}, \quad \iint_{R} \frac{1}{r^{2}(1-r)^{n-2}} \sum_{k=1}^{n-1} \sum_{j=1}^{n-1} \frac{\partial u}{\partial \theta_{j}} \theta_{j} \theta_{k} \frac{\partial k}{\partial \theta_{k}}
$$

and

$$
\iint_{R} \frac{1}{(1-r)^{n-1}} \sum_{j=1}^{n-1} \frac{\partial u}{\partial \theta_{j}} \bar{\theta}_{j} P(\bar{\theta})
$$

are left to deal with. These will be replaced by dyadic martingales.

First each integral is written as a sum of smaller parts:

$$
\iint_{R} \frac{1}{r^{2}(1-r)^{n-2}} \sum \frac{\partial u}{\partial \theta_{i}} \frac{\partial k}{\partial \theta_{i}}=\sum_{i=1}^{n-1} \sum_{Q \in \mathcal{Q}} \iint_{T_{Q}} \frac{1}{r^{2}(1-r)^{n-2}} \frac{\partial u}{\partial \theta_{i}} \frac{\partial k}{\partial \theta_{i}}
$$

where $\mathcal{Q}$ is the collection of all dyadic subcubes $Q \subseteq P\left(3 Q_{0}\right) \subseteq S_{0}$ with $\operatorname{supp} k_{1-r}\left(\theta_{x}-\cdot\right) \cap Q \neq \emptyset$. The regions $T_{Q}$ are segments of spherical shells that 
are obtained by projecting $Q$ radially into the region $c / 2^{k+1}<1-r<c / 2^{k}$; here $c$ depends on $\beta$ and $r_{0}$, and $\ell\left(\widehat{P}^{-1}(Q)\right)=1 / 2^{k}$.

Now each $\sum_{Q \in \mathcal{Q}} \lambda_{Q}$ is further subdivided into sums

$$
\sum_{i=1}^{m} \sum_{Q \in \mathcal{Q}^{i}} \lambda_{Q}=\sum_{i=1}^{m} \Lambda_{\varepsilon, k}^{x_{i}},
$$

where $\mathcal{Q}^{i}=\mathcal{Q}^{x_{i}}$ is a collection of cubes from $\mathcal{Q}$. These cubes are chosen so that if $Q \in \mathcal{Q}^{i}$ then there is a dyadic cube $Q^{\prime \prime} \in \mathcal{Q}$ so that $3 Q \subseteq Q^{\prime \prime}+x_{i}$ and $\ell\left(\widehat{P}^{-1}\left(Q^{\prime \prime}\right)\right)=2^{3} \ell\left(\widehat{P}^{-1}(Q)\right)$. The construction of the $\mathcal{Q}^{i}$ is accomplished in $\mathbb{R}^{n-1}$, dealing with the (truly) dyadic cubes $\widehat{P}^{-1}(Q)$ that were originally used to define the "dyadic" surface cubes. The following Lemma 3 guarantees that such a subdivision of $\mathcal{Q}$ exists:

Lemma 3 ([CWW], [BM]). There are a finite set of points $\left\{x_{i}\right\}_{i=1}^{M}$ in $\mathbb{R}^{n-1}$ and a disjoint collection $\mathcal{Q}^{x_{i}}$ of cubes so that

(i) $\bigcup_{i=1}^{M} \bigcup_{Q \in \mathcal{Q}^{x_{i}}} \widehat{P}^{-1}(Q)=\bigcup_{Q \in \mathcal{Q}} \widehat{P}^{-1}(Q)=\widehat{P}^{-1}\left(3 Q_{0}\right)$.

(ii) For each $Q \in \mathcal{Q}^{x_{i}}$ there is a cube $Q^{\prime \prime} \in \mathcal{Q}$ so that $\ell\left(\widehat{P}^{-1}\left(Q^{\prime \prime}\right)\right)=$ $2^{3} \ell\left(\widehat{P}^{-1}(Q)\right)$ and $\widehat{P}^{-1}(3 Q) \subseteq \widehat{P}^{-1}\left(Q^{\prime \prime}+\theta_{x_{i}}\right)=\widehat{P}^{-1}\left(Q^{\prime \prime}\right)+x_{i}$.

(iii) If $Q_{1}, Q_{2} \in \mathcal{Q}^{x_{i}}$ and $Q_{1} \neq Q_{2}$, then $Q_{1}^{\prime \prime} \neq Q_{2}^{\prime \prime}$.

The lemma is proved exactly as in [CWW] dealing with the $\widehat{P}^{-1}(Q)$, $\widehat{P}^{-1}\left(Q^{\prime \prime}\right)$ and the proof is consequently omitted.

There are three crucial properties that hold for the integrals $\lambda_{Q}=$ $\int_{T_{Q}} I_{j}\left(\theta_{x}-\theta_{y}\right) d r d \theta_{y}$ and these properties are the ones that allow the martingale square functions to be bounded by $c A_{\gamma} f$. They are

$$
\begin{gathered}
\operatorname{supp} \lambda_{Q} \subseteq 3 Q, \quad \int_{S_{0}} \lambda_{Q}=0, \\
\left\|\lambda_{Q}\right\|_{\operatorname{Lip} \alpha} \ell(Q)^{\alpha} \leq c\left(\int_{T_{Q}}|\nabla u|^{2}(1-r)^{2-n}\right)^{1 / 2} .
\end{gathered}
$$

The first property of dyadic cubes in $S^{n-1}$ means supp $\lambda_{Q} \subseteq \widehat{P}(3 \widehat{Q})$ if $\widehat{Q} \subseteq \mathbb{R}^{n-1}$ and $Q=\widehat{P}(\widehat{Q})$. To have this hold one needs $\operatorname{supp} k_{1-r}\left(\theta_{x}-\theta_{y}\right)$ $\subseteq Q$ together with all neighboring dyadic surface cubes of $Q$. For this the distortion produced by the projection operator $\widehat{P}$ must be taken into account. This is accomplished by taking

$$
\operatorname{supp} k(\theta)=\operatorname{supp} P(\theta) \subseteq\left\{\theta \in S_{0}:|\theta-(0, \ldots, 0,-1)|<\beta\right\},
$$

where $\beta=r_{0} \gamma /\left(r_{0}+12 \sqrt{n-1}\right)$. This choice of $\beta$ in relation to $\gamma$ is also designed for the proof of Theorem 3 below. Here $r_{0}$ is a constant such that 
$r_{0} \ell\left(\widehat{P}^{-1}(Q)\right)<\ell(Q)<\left(1 / r_{0}\right) \ell\left(\widehat{P}^{-1}(Q)\right)$ for all cubes $Q \in \mathcal{Q}$ and

$$
T_{Q}=\left\{(r, \theta): \theta \in Q, \frac{\ell\left(\widehat{P}^{-1}(Q)\right)}{2 \beta}<1-r \leq \frac{\ell\left(\widehat{P}^{-1}(Q)\right)}{\beta}\right\}
$$

for any $Q \in \mathcal{Q}$.

The regions $T_{Q}$ with $\ell\left(\widehat{P}^{-1}(Q)\right)=\ell\left(\widehat{P}^{-1}\left(Q_{0}\right)\right) / 2^{k}$ are not of uniform volume. However, for $Q_{1}, Q_{2}$ with $\ell\left(\widehat{P}^{-1}\left(Q_{1}\right)\right)=\ell\left(\widehat{P}^{-1}\left(Q_{2}\right)\right),\left|T_{Q_{1}}\right| \sim\left|T_{Q_{2}}\right|$ with constants depending on the function $\widehat{P}(x)$. Also the $T_{Q}$ cover a region in the lower hemisphere of $B_{1}(0)$ so for any $\varepsilon>0$ the region $R$ in Lemma 1 will be contained in $\bigcup_{Q \in \mathcal{Q}} T_{Q}$.

Now,

$$
\Lambda_{\varepsilon, k}^{i}\left(\theta_{x}\right)=\sum_{Q \in \mathcal{Q}^{i}} \lambda_{Q}\left(\theta_{x}\right)=\sum_{\ell(Q) \geq 1 / 2^{m+2}}\left(\lambda_{Q}+\lambda_{Q, \varepsilon}\right),
$$

where $1 / 2^{m+3}<\varepsilon \leq 1 / 2^{m+2}$ and $\lambda_{Q, \varepsilon}=\int_{T_{Q} \cap\{r>1-\varepsilon\}} I_{k}\left(\theta_{x}\right)$ (if $\varepsilon=1 / 2^{m+2}$, $\lambda_{Q, \varepsilon}$ disappears). By using the shifted grid of cubes in $\mathcal{Q}^{i}, \Lambda_{\varepsilon, k}^{i}$ can be approximated by the function $E\left(\Lambda_{\varepsilon, k}^{i} \mid \mathcal{F}_{m-1}\right)$, the conditional expectation of $\Lambda_{\varepsilon, k}^{i}$ with respect to the $\sigma$-algebra $\mathcal{F}_{m-1}$. Notice that

$$
E\left(\Lambda_{\varepsilon, k}^{i} \mid \mathcal{F}_{m-1}^{i}\right)=E\left(\Lambda_{1 / 2^{m+2}, k}^{i} \mid \mathcal{F}_{m-1}^{i}\right)
$$

because $\int_{Q_{m-1}} \lambda_{Q, \varepsilon}=0$ for any $Q_{m-1}$ in the generating set for $\mathcal{F}_{m-1}^{i}$. This happens because $\operatorname{supp} \lambda_{Q, \varepsilon} \subseteq$ some $Q_{m-1} \in \mathcal{F}_{m-1}^{i}$ by Lemma 3 .

Now, with $\sum_{Q}^{\prime}:=\sum_{c\left(\delta_{0}\right) \geq \ell\left(\widehat{P}^{-1}(Q)\right)>1 / 2^{m+2}}$,

$$
\begin{aligned}
\left|\Lambda_{\varepsilon, k}^{i}\left(\theta_{x}\right)-E\left(\Lambda_{\varepsilon, k}^{i} \mid \mathcal{F}_{m-1}^{i}\right)\right| & \left|\sum_{Q}^{\prime} \lambda_{Q}^{i}-\frac{1}{\left|Q_{m-1}\right|} \int_{Q_{m-1}} \sum_{Q}^{\prime} \lambda_{Q}^{i}\right| \\
\leq & \sum_{Q}^{\prime} \frac{1}{\left|Q_{m-1}\right|} \int_{Q_{m-1}}\left|\lambda_{Q}^{i}\left(\theta_{x}\right)-\lambda_{Q}^{i}\left(\theta_{z}\right)\right| d \theta_{z} \\
\leq & \sum_{Q}^{\prime} \frac{1}{\left|Q_{m-1}\right|} \int_{Q_{m-1}} \int_{T_{Q}} \frac{1}{r^{2}(1-r)^{n-2}}\left|\frac{\partial u}{\partial \theta_{j}}\left(r \theta_{w}\right)\right| \\
& \cdot\left|\frac{\partial k}{\partial \theta_{j}}\left(\frac{\theta_{x}-\theta_{w}}{1-r}\right)-\frac{\partial k}{\partial \theta_{j}}\left(\frac{\theta_{z}-\theta_{w}}{1-r}\right)\right| d r d \theta_{w} d \theta_{z} \\
\leq & \sum \frac{1}{\left|Q_{m-1}\right|} \iint_{Q_{m-1}} \frac{1}{T_{Q} r^{2}(1-r)^{n-1}}\left|\frac{\partial u}{\partial \theta_{j}}\left(r \theta_{w}\right)\right| \\
& \cdot\left|\nabla_{\bar{\theta}} \frac{\partial k}{\partial \theta_{j}}\left(\theta^{*}\right)\right| \cdot\left|\frac{\theta_{x}-\theta_{z}}{1-r}\right| d r d \theta_{w} d \theta_{z}
\end{aligned}
$$




$$
\begin{aligned}
& \leq C \sum \int_{T_{Q}} \frac{1}{(1-r)^{n}}\left|\nabla_{\theta} u\right| \sup _{Q_{m-1}}\left|\theta_{x}-\theta_{z}\right| \\
& \leq C \sum \ell\left(Q_{m-1}\right)\left(\int_{T_{Q}}\left|\nabla_{\theta} u\right|^{2}(1-r)^{2-n}\right)^{1 / 2}\left(\int_{T_{Q}} \frac{1}{(1-r)^{n+2}}\right)^{1 / 2} \\
& \leq C\left(\sum_{Q}^{\prime} \int_{T_{Q}}\left|\nabla_{\theta} u\right|^{2}(1-r)^{2-n}\right)^{1 / 2}\left(\sum_{Q}^{\prime}\left(\frac{\ell\left(Q_{m-1}\right)}{\ell(Q)}\right)^{2}\right)^{1 / 2}=C A_{\gamma}^{\delta} f\left(\theta_{x}\right) .
\end{aligned}
$$

The last inequality follows because the cone $\Gamma_{\gamma}^{\delta}\left(\theta_{x}\right)$ contains any $T_{Q}$ where $\operatorname{supp} \lambda_{Q}^{i} \cap Q_{m-1} \neq \emptyset$ and $\theta_{x} \in Q_{m-1}$. Taking $\gamma=\left(1+12 \sqrt{n-1} / r_{0}\right) \beta$ is enough to guarantee this.

A similar calculation shows that each integrand in $\Lambda_{\varepsilon, k}, k=4,5,6$, has the same upper bound for $\left|\Lambda_{\varepsilon, k}^{i}\left(\theta_{x}\right)-E\left(\Lambda_{\varepsilon, k}^{i} \mid \mathcal{F}_{m-1}^{i}\right)\right|, i=1, \ldots, M$.

The last result of Part I is Theorem 3. To see that the dyadic martingale square function for the dyadic martingale $\left\{E\left(\Lambda_{\varepsilon, k}^{i} \mid \mathcal{F}_{m-1}^{i}\right)\right\}_{m=1}^{\infty}$ can be bounded by $C\left\|A_{\gamma}^{\delta} f\right\|_{\infty}$ a.e., it is helpful to notice that for a fixed $Q \in \mathcal{Q}^{i}$ if $Q_{m-1} \supseteq 3 Q$ and $\ell\left(\widehat{P}^{-1}\left(Q_{m-1}\right)\right)=2^{3} \ell(Q)$, then $f_{Q_{m-1}} \lambda_{Q}^{i}=0$ so $d_{j} \chi_{Q_{j-1}}=0$ if $j>m$. When $j=m$, we have

$$
\left\|d_{m} \chi_{Q_{m-1}}\right\|_{\infty}=\sup _{Q_{m} \subseteq Q_{m-1}}\left|f_{Q_{m}} \lambda_{Q}^{i}-f_{Q_{m-1}} \lambda_{Q}^{i}\right|
$$

if $\theta_{x} \in Q_{m-1}$. The estimate for this term in the dyadic square function only involves $\nabla u\left(r \theta_{y}\right)$ for $r \theta_{y} \in T_{Q}$ and the most extreme case will be that $Q$ lies in the $2^{n}$ th section of $Q_{m-1}, Q_{m}$, that is farthest from $\theta_{x}$. Then at most the region in $\left\|d_{m} \chi_{Q_{m-1}}\right\|_{\infty}$ will involve 5 cubes beyond the cube $Q$ such that $\theta_{x} \in \operatorname{supp} T_{Q}$, i.e. at a distance $6 \sqrt{n-1}\left(1 / r_{0}\right) \ell\left(\widehat{P}^{-1}(Q)\right)$, so one needs $\gamma \geq \beta\left(1+12 \sqrt{n-1} / r_{0}\right)$.

THEOREM 3. For $\Lambda_{k}^{i}\left(\theta_{x}\right)$ the martingale limit function of the dyadic martingale

$$
\left\{E\left(\Lambda_{1 / 2^{m+1}, k}^{i} \mid \mathcal{F}_{m-1}^{i}\right)\right\}_{m=1}^{\infty},
$$

with $\Lambda_{\varepsilon, k}^{i}$ defined above, the dyadic martingale square function satisfies $S \Lambda_{k}^{i}\left(\theta_{x}\right) \leq C A_{\gamma}^{\delta} f\left(\theta_{x}\right)$ with $C$ independent of $\theta_{x}$ and $f$.

Proof. Notice that it is obvious that $\left\{E\left(\Lambda_{1 / 2^{m+1}, k}^{i} \mid \mathcal{F}_{m-1}^{i}\right)\right\}_{m=1}^{\infty}$ is a dyadic martingale. The rest of the proof will actually show that each dyadic square function $S_{m}$ is uniformly bounded, with constant independent of $m$. This implies that the family of martingale functions is uniformly integrable and so has an $L^{1}$ limit function $\Lambda_{\varepsilon, k}^{i}$. Actually the uniform bound on $S_{m}$ is 
enough to prove Theorem 1. Now with $\sum_{Q}^{\prime}$ as in the previous proof,

$$
\begin{aligned}
& S^{2} \Lambda^{i}\left(\theta_{x}\right)=\sum_{Q_{j} \in \mathcal{F}^{i}}\left\|f_{Q_{j}} \sum \lambda_{Q}-f_{Q_{j-1}} \sum \lambda_{Q}\right\|_{\infty}^{2} \\
& =\sum_{Q_{j} \in \mathcal{F}^{i}}\left\|\sum_{Q}^{\prime}\left(f_{Q_{j}} \lambda_{Q}-\underset{Q_{j-1}}{f} \lambda_{Q}\right)\right\|_{\infty}^{2} \\
& \leq \sum_{Q_{j} \in \mathcal{F}^{i}}\left\|\sum_{Q}^{\prime} f_{Q_{j}} f_{Q_{j-1}}\left(\lambda_{Q}\left(\theta_{w}\right)-\lambda_{Q}\left(\theta_{z}\right)\right) d \theta_{z} d \theta_{w}\right\|_{\infty}^{2} \\
& \leq \sum_{Q_{j} \in \mathcal{F}^{i}}\left(\sum_{Q}^{\prime} f_{Q_{j}} f_{Q_{j-1}}\left\|\lambda_{Q}\right\|_{\operatorname{Lip} \alpha} \ell\left(Q_{j-1}\right)^{\alpha} d \theta_{z} d \theta_{w}\right)^{2} \\
& =c \sum_{Q_{j} \in \mathcal{F}^{i}} \ell\left(Q_{j-1}\right)^{2 \alpha}\left(\sum\left\|\lambda_{Q}\right\|_{\operatorname{Lip} \alpha} \frac{\ell(Q)^{\beta}}{\ell(Q)^{\beta}}\right)^{2} \\
& \leq C \sum_{Q_{j} \in \mathcal{F}^{i}} \ell\left(Q_{j-1}\right)^{2 \alpha}\left(\sum_{Q}^{\prime} \frac{1}{\ell\left(\widehat{P}^{-1}(Q)\right)^{2 \beta}}\right)\left(\sum\left\|\lambda_{Q}\right\|_{\operatorname{Lip} \alpha}^{2} \ell(Q)^{2 \beta}\right) \\
& =C \sum_{Q_{j} \in \mathcal{F}^{i}} \ell\left(Q_{j-1}\right)^{2 \alpha} \cdot \ell\left(Q_{j-1}\right)^{-2 \beta} \sum_{Q}^{\prime}\left\|\lambda_{Q}\right\|_{\operatorname{Lip} \alpha, Q_{j-1}}^{2} \ell(Q)^{2 \beta} \\
& \leq C \sum_{Q \in \mathcal{Q}}\left\|\lambda_{Q}\right\|_{\operatorname{Lip} \alpha}^{2} \ell(Q)^{2 \beta} . \sum_{\ell\left(Q_{j-1}\right) \leq 2^{3} \ell(Q)} \ell\left(Q_{j-1}\right)^{2(\alpha-\beta)} .
\end{aligned}
$$

Here $T_{Q}$ is in the sum of $\lambda_{Q}$ that make up $\Lambda^{i}$, and $\theta_{x}$ is near $T_{Q}$ as described above. This means

$$
\begin{aligned}
S^{2} \Lambda^{i}\left(\theta_{x}\right) & \leq C \sum_{Q}\left\|\lambda_{Q}\right\|_{\operatorname{Lip} \alpha}^{2} \ell(Q)^{2 \alpha} \\
& \leq C \sum_{Q} \int_{T_{Q}}|\nabla u|^{2} \cdot(1-r)^{2-n} \leq C A_{\gamma}^{\delta_{0}} f\left(\theta_{x}\right) .
\end{aligned}
$$

It is useful to extend the class of operators $L_{0}$ for the application of Theorem 4 . Note that Theorem 1 is also valid for any strictly elliptic constant coefficient operator. Basically the same proof works for solutions to these operator equations. If $L=\sum_{i, j=1}^{n} \frac{\partial}{\partial x_{i}}\left(a_{i j} \frac{\partial}{\partial x_{j}}\right)$ in rectangular coordinates, then in spherical coordinates

$$
\begin{aligned}
L= & \left(\sum_{i, j=1}^{n} a_{i j} \theta_{i} \theta_{j}\right) \frac{\partial^{2}}{\partial r^{2}}-\frac{1}{r^{2}}\left(\sum_{j<n, i=1}^{n} a_{i j} \theta_{i} \frac{\partial}{\partial \theta_{j}}+\sum_{i<n, j=1}^{n} a_{i j} \theta_{j} \frac{\partial}{\partial \theta_{i}}\right) \\
& +\frac{1}{r}\left(\sum_{j<n, i=1}^{n} a_{i j} \theta_{i} \frac{\partial}{\partial r \partial \theta_{j}}+\sum_{i<n, j=1}^{n} a_{i j} \theta_{j} \frac{\partial^{2}}{\partial r \partial \theta_{i}}\right)
\end{aligned}
$$




$$
\begin{aligned}
& -\frac{2}{r}\left(\sum_{i, j=1}^{n} a_{i j} \theta_{i} \theta_{j}\right)\left(\sum_{l=1}^{n-1} \theta_{l} \frac{\partial^{2}}{\partial r \partial \theta_{l}}\right)+\frac{1}{r^{2}}\left(\sum_{i, j=1}^{n-1} a_{i j} \frac{\partial^{2}}{\partial \theta_{i} \partial \theta_{j}}\right) \\
& -\frac{1}{r^{2}}\left(\sum_{j<n, i=1}^{n} a_{i j} \theta_{i}\left(\sum_{l=1}^{n-1} \theta_{l} \frac{\partial^{2}}{\partial \theta_{l} \partial \theta_{j}}\right)+\sum_{i<n, j=1}^{n} a_{i j} \theta_{j}\left(\sum_{l=1}^{n-1} \theta_{l} \frac{\partial^{2}}{\partial \theta_{l} \partial \theta_{i}}\right)\right) \\
& +\frac{1}{r^{2}}\left(\sum_{i, j=1}^{n} a_{i j} \theta_{i} \theta_{j}\right)\left(\sum_{l=1}^{n-1} \sum_{k=1}^{n-1} \theta_{k} \theta_{l} \frac{\partial^{2}}{\partial \theta_{k} \partial \theta_{l}}\right)+\frac{1}{r}\left(\sum_{i=1}^{n} a_{i i}-\sum_{i, j=1}^{n} a_{i j} \theta_{i} \theta_{j}\right) \frac{\partial}{\partial r} \\
& +\frac{3}{r^{2}}\left(\sum_{i, j=1}^{n} a_{i j} \theta_{i} \theta_{j}\right)\left(\sum_{l=1}^{n-1} \theta_{l} \frac{\partial}{\partial \theta_{l}}\right)-\frac{1}{r^{2}}\left(\sum_{i=1}^{n} a_{i i}\right)\left(\sum_{l=1}^{n-1} \theta_{l} \frac{\partial}{\partial \theta_{\ell}}\right) .
\end{aligned}
$$

The fact that the $a_{i j}$ are constant and satisfy $\lambda|\xi|^{2} \leq \sum_{i, j=1}^{n} \xi_{i} a_{i j} \xi_{j}$ $\leq \lambda^{-1}|\xi|^{2}$ means that one can substitute for $\partial^{2} u / \partial r^{2}$ as in the proof of Theorem 1. The formula is much longer; however the same kinds of estimations will work here to give the exponential square result, using direct estimation on the drift terms and integration by parts along with replacement by martingales on the principal order terms. To estimate the boundary terms the device of averaging replaces using Lemma 2 ([CS1]).

II. In this part of the paper $S_{\alpha} u(\theta)=\left(\int_{\Gamma_{\alpha}(\theta)}|\nabla u(y)|^{2} \delta(y)^{2-n} d y\right)^{1 / 2}$ is used to devoted the Lusin area integral of $u(y)$ over the cone $\Gamma_{\alpha}(\theta)=\Gamma_{\alpha}^{\delta}(\theta)$, a Euclidean cone as defined in the Appendix. The following result will be established:

THEOREM 4. For $L_{0}, L_{1}$ strictly elliptic divergence form operators with coefficients satisfying the condition (Cc) below, suppose the solutions to $L_{0} u_{0}=0$ in $B_{1}(0)$ satisfy the exponential square theorem. Then solutions $u$ to $L_{1} u_{1}=0$ in $B_{1}(0)$ also satisfy the EST.

DEFinition (EST). For $L$ a strictly elliptic divergence form operator on $B_{1}(0)$, a solution to $L u=0$ on $B_{1}(0)$ satisfies the exponential square theorem if $S u \in L^{\infty}\left(\partial B_{1}(0)\right)$ implies that $\left.u\right|_{\partial B}=f$ lies in the exponential square class.

(Cc) For all $q \in \partial B_{1}(0)$ and $0<r<r_{0}$ there is a coefficient $\varepsilon(r)$ so that

$$
\left(\frac{1}{\omega_{0}\left(\Delta_{r}(q)\right)} \int_{T_{r}(q)} \frac{a(y)^{2}}{\delta(y)^{2}} G_{0}(0 ; y) d y\right) \leq \varepsilon(r)
$$

with $\varepsilon(r) \rightarrow 0$ as $r \rightarrow 0$ and in fact $\varepsilon\left(2^{j} r\right) \leq 2^{\gamma j} r^{\gamma}$ where $\gamma<\alpha$, $\alpha$ is the coefficient of Hölder continuity at $\partial B_{1}(0)$ for solutions of $L_{0} u=0$ that vanish locally on $\partial B_{1}(0)$. 
Here $\omega_{0}=\omega_{0}^{0}$ is the elliptic measure associated with $L_{0} ; \omega_{0}^{x}$ is this measure evaluated at the point $x$ in $B_{1}(0) ; G_{0}(x ; y)$ is the Green function for $L_{0} ; \Delta_{r}(q)=B_{r}(q) \cap \partial B_{1}(0)$ and $T_{r}(q)=B_{r}(q) \cap B_{1}(0)$ for $q \in \partial B_{1}(0)$; $\delta(y)=\operatorname{dist}\left(y, \partial B_{1}(0)\right)$ and $a(y)=\sup _{x \in B_{\delta(y) / 2}(y)}|\varepsilon(x)|$, where $\varepsilon(x)=$ $\sup _{i, j}\left|\varepsilon_{i j}(x)\right|$ and $\varepsilon_{i j}(x)=b_{i j}(x)-a_{i j}(x)$. Moreover, the operators

$$
L_{0}=\sum_{i, j=1}^{n} \frac{\partial}{\partial x_{i}}\left(a_{i j}(x) \frac{\partial}{\partial x_{j}}\right), \quad L_{1}=\sum_{i, j=1}^{n} \frac{\partial}{\partial x_{i}}\left(b_{i j}(x) \frac{\partial}{\partial x_{j}}\right)
$$

satisfy $\lambda^{-1}|\xi|^{2} \leq \xi_{i} C_{i j} \xi_{j} \leq \lambda|\xi|^{2}$ for $C_{i j}=a_{i j}$ and $b_{i j}$ and for some $\lambda>0$.

Definition. $f \in \exp L^{2}(d \mu)$ if for any surface "cube" $Q \subseteq \partial B_{1}(0)$ there are constants $C_{1}, C_{2}>0$ independent of $Q$ so that

$$
\left(\frac{1}{\mu(Q)} \int_{Q} \exp \left\{C_{1}\left|f-f_{\mu(Q)}\right|^{2}\right\} d \mu(y)\right) \leq C_{2}<\infty,
$$

where $f_{\mu(Q)}=\mu(Q)^{-1} \int_{Q} f(y) d \mu(y)$.

In this paper $\mu$ will be taken to be surface (Lebesgue) measure on $\partial B_{1}(0)$, and the elliptic measure associated to $L_{0}$ will be assumed to satisfy $d \omega_{0} \in$ $B^{2}(d \mu)$, i.e.

$$
\left(\frac{1}{\sigma(Q)} \int_{Q}\left(\frac{d \omega_{0}}{d \sigma}(x)\right)^{2} d \sigma(x)\right)^{1 / 2} \precsim \frac{\omega_{0}(Q)}{\sigma(Q)} .
$$

The measure in Theorem 4 and in the EST will be surface measure. It is crucial that the results of $[\mathrm{FKP}]$ hold for the measure $d \omega_{1}$, given the Carleson condition $(\mathrm{Cc})$.

In order to prove that the Carleson condition with vanishing trace is sufficient to guarantee that the exponential square theorem will hold for solutions of one operator whenever it holds for solutions of a given operator, one can obtain a pointwise comparison of area integrals. This is done by using arguments similar to those in $[\mathrm{FKP}]$; the solution $u_{1}$ behaves like a perturbation of $u_{0}$ when the coefficients satisfy $(\mathrm{Cc})$.

One reason for proving Theorem 1 in Part I was to guarantee that Theorem 4 is not vacuous. Theorem 1 shows that for $L_{0}=\Delta$, the EST is true; so Theorem 4 then shows that EST will also hold for solutions to $L_{1} u_{1}=0$ when $L_{1}$ is a mild perturbation of $\Delta$ (or of any strictly elliptic constant coefficient operator). This result extends the class of elliptic operators for which the EST is known to be valid. It is a start towards proving an EST for solutions to more general elliptic operators in the upper half-plane. In [CS1] a result for a restricted class of elliptic operators was established for the upper half-plane. The coefficients were assumed to match the Laplacian in the vertical direction, i.e. $a_{n j}=\delta_{n j}, j=1, \ldots, n$. Theorem 4 removes 
this restriction, replacing it by the broader Carleson condition. R. Bañuelos and $\mathrm{C}$. Moore $[\mathrm{BM}]$ note that an exponential square result for any operator $L$ on $\mathbb{R}_{+}^{n+1}$ whose pull-back by a Lipschitz map is $\Delta$ would open the way for an easy proof of good- $\lambda$ inequalities for the nontangential maximal function and the Lusin area integral of a harmonic function with sharp constants. Theorem 4 does not accomplish this much, but it is a step in that direction.

There are other facts that can be deduced from the proof of Theorem 4 . The comparison of area integrals obtained in the Main Lemma below may be of interest in itself. As a major step in proving the Main Lemma the key estimate

$$
\left(f_{R_{j}^{*}}|F(y)|^{2} d y\right)^{1 / 2} \precsim \varepsilon\left(\ell\left(R_{j}\right)\right) M_{\omega_{0}} S_{\beta}\left(u_{1}\right)\left(q_{0}\right)
$$

is shown to be valid for any fixed dilation $R_{j}^{*}$ of a Whitney type region $R_{j} \subseteq B_{1}(0)$. Here $F(y)=u_{1}(y)-u_{0}(y), \ell\left(R_{j}\right) \simeq \operatorname{diam} R_{j}^{*} \simeq \operatorname{dist}\left(R_{j}^{*}, \partial B_{1}(0)\right)$, $q_{0}$ is any point on $\partial B_{1}(0)$ that lies in the radial projection of $R_{j}$ onto $\partial B_{1}(0)$, $\varepsilon\left(\ell\left(R_{j}\right)\right)$ is the Carleson coefficient and

$$
M_{\omega_{0}} g(q)=\sup _{\Delta_{r}(q)} \frac{1}{\omega_{0}\left(\Delta_{r}(q)\right)} \int_{\Delta_{r}(q)}|g(\widehat{q})| d \omega_{0}(\widehat{q})
$$

is the Hardy-Littlewood maximal function with respect to the elliptic measure $\omega_{0}$ of $L_{0}$.

It is worth noting that the above key estimate for $\left(f_{R_{j}^{*}}|F(y)|^{2} d y\right)^{1 / 2}$ can be used to prove the good- $\lambda$ inequality in [FKP, Lemma 2.16]. With suitable modifications this condition and proof also give the good- $\lambda$ inequality in [CS2, Lemma 7].

Let

$$
M\left(q_{0}\right)=\max \left(M_{\omega_{0}} S_{\beta} u_{1}\left(q_{0}\right),\left[M_{\omega_{0}} S_{\beta} u_{1}\left(q_{0}\right)\right]^{1 / 2}\right) .
$$

Theorem 4 will follow from the

MAIn Lemma. Suppose $L_{0} u_{0}=0=L_{1} u_{1}$ in $B_{1}(0)$, where $L_{0}$ and $L_{1}$ are as in Theorem 4. If $\left.u_{1}\right|_{\partial B_{1}(0)}=f=\left.u_{0}\right|_{\partial B_{1}(0)}$, where $f \in \operatorname{BMO}\left(\partial B_{1}, d \omega_{0}\right)$, then there is a constant $C=C\left(\lambda, n,\|f\|_{\mathrm{BMO}}, \alpha, \beta\right)$ so that

$$
S_{\alpha} u_{0}(q) \leq C M(q) \quad \text { for } d \omega_{0} \text { a.e. } q \in \partial B_{1}(0) .
$$

Before proving the Main Lemma, it is easy to see that if $S_{\beta} u_{1} \in$ $L^{\infty}\left(\partial B_{1}, d \omega_{0}\right)$ then

$$
M_{\omega_{0}} S_{\beta} u_{1}(q) \leq\left\|S_{\beta} u_{1}\right\|_{\infty, \omega_{0}} \quad \text { for } d \omega_{0} \text { a.e. } q .
$$

The Main Lemma says that if $S_{\beta} u_{1} \in L^{\infty}\left(\partial B_{1}, d \omega_{1}\right)$ then $S_{\alpha} u_{0} \in$ $L^{\infty}\left(\partial B_{1}, d \omega_{0}\right)$ and by the hypothesis of Theorem $4, u_{0}$ satisfies EST, so $f \in \exp L^{2}\left(\partial B_{1}\right)$. The reason that the assumption $f \in \operatorname{BMO}\left(\partial B_{1}, d \omega_{0}\right)$ is 
not needed for Theorem 4 is the following: the fact that the coefficients of $L_{0}$ and $L_{1}$ satisfy (Cc) means that the $B^{2}$ result of $[\mathrm{FKP}]$ is valid so $\omega_{0} \in B^{2}(\sigma)$ $\Rightarrow \omega_{1} \in B^{2}(\sigma)$. This fact is enough to guarantee that

$$
S_{\beta} u_{1} \in L^{\infty}\left(\partial B_{1}, d \omega_{1}\right) \Rightarrow f \in \operatorname{BMO}\left(d \omega_{0}\right),
$$

because one can use the following theorem of C. Kenig ([K, Chapter 1, Theorem 1.5.18], quoted below for clarity) to see

$$
S_{\beta} u_{1} \in L^{\infty}\left(\partial B_{1}, d \omega_{1}\right) \Rightarrow f \in \operatorname{BMO}\left(d \omega_{1}\right) .
$$

Then $d \omega_{1} \in B^{2}\left(d \omega_{0}\right) \Rightarrow d \omega_{0} \in B^{q}\left(d \omega_{1}\right)$ for some $q>1$ so this is sufficient to have $f \in \operatorname{BMO}\left(d \omega_{0}\right)$.

Theorem (C. Kenig). Let $L u=0$ in $B_{1}(0)$. Then

$$
u(x)=\int_{\partial B_{1}(0)} k(x ; q) f(q) d \omega(q)
$$

with $f \in \operatorname{BMO}\left(\partial B_{1}, d \omega\right)$ if and only if

$$
\frac{1}{\omega\left(\Delta_{r}(q)\right)} \int_{T_{r}(q)} G(0 ; y)|\nabla u(y)|^{2} d y \leq C .
$$
$S^{n-1}$,

If $S_{\beta} u \in L^{\infty}(d \omega)$ then the basic estimate, for $y^{*}$ the projection of $y$ onto

$$
G(0 ; y) \simeq \frac{\omega\left(\Delta_{\delta(y)}\left(y^{*}\right)\right)}{\delta(y)^{n-2}}
$$

shows that

$$
\begin{gathered}
\frac{1}{\omega\left(\Delta_{r}\right)} \int_{T_{r}} G(0 ; y)|\nabla u(y)|^{2} d y \leq \frac{1}{\omega\left(\Delta_{r}\right)} \int_{T_{r}} \frac{\omega\left(\Delta_{\delta(y)}\left(y^{*}\right)\right)}{\delta(y)^{n-2}}|\nabla u(y)|^{2} d y \\
\underset{\text { Fubini }}{\precsim} \frac{1}{\omega\left(\Delta_{r}\right)} \int_{\Delta_{r}} \int_{\Gamma_{\beta}^{\delta_{0}}(q)}|\nabla u(y)|^{2} \delta(y)^{2-n} d y d \omega(q) \\
=\frac{1}{\omega\left(\Delta_{r}\right)} \int_{\Delta_{r}} S_{\beta} u(q) d \omega(q) \leq\left\|S_{\beta} u\right\|_{\infty} .
\end{gathered}
$$

So $S_{\beta} u_{1} \in L^{\infty}\left(\partial B_{1}, d \omega_{1}\right) \Rightarrow f \in \operatorname{BMO}\left(\partial B_{1}, d \omega_{1}\right)$.

To prove the Main Lemma, an argument similar to that of [FKP] in their proof of Lemma 2.9 can be employed. Here, however, all estimates will be kept local, both for $f_{R_{j}}|\delta(y) \nabla F(y)|^{2} d y$ and for $\left(f_{R_{j}}|F(y)|^{2} d y\right)^{1 / 2}$ until the very end of the proof. Then instead of taking sups, the local estimates will be added to show $S_{\alpha} F(q) \precsim M(q)$ for a.e. $q \in \partial B_{1}(0)$.

It is easy to see this implies $S_{\alpha} u_{0}(q) \leq c^{\prime} M(q)$ also. Start with the inequality 


$$
\begin{aligned}
& f_{R_{j}}|\delta(y) \nabla F(y)|^{2} d y \leq \frac{2}{\ell\left(R_{j}\right)} \int_{\ell\left(R_{j}\right)}^{3 \ell\left(R_{j}\right) / 2}\left(f_{R_{j}}|\delta \nabla F|^{2}\right) d x \\
& \quad \lesssim \frac{\delta_{j}^{2}}{\left|R_{j}\right|}\left(\frac{1}{\delta_{j}} \int_{N\left(\partial R_{j}\right)}|F(y)||A \nabla F(y) \cdot \vec{n}|+\frac{1}{\delta_{j}} \int_{N\left(\partial R_{j}\right)}|F||\varepsilon|\left|\nabla u_{1} \cdot \vec{n}\right|\right. \\
& \left.+\int_{R_{j}^{*}}|\vec{\nabla} F|\left|\varepsilon \vec{\nabla} u_{1}\right|\right) \leq C(\lambda, n)\left(f_{R_{j}^{*}}|F|^{2}\right)^{1 / 2}\left(f_{R_{j}^{*}}|\delta \nabla F|^{2}\right)^{1 / 2} \\
& +\varepsilon_{j}\left(f_{R_{j}^{*}}|F|^{2}\right)^{1 / 2}\left(f_{R_{j}^{*}}\left|\delta \nabla u_{1}\right|^{2}\right)+\varepsilon_{j}\left(f_{R_{j}^{*}}|\delta \nabla F|^{2}\right)^{1 / 2} \cdot\left(f_{R_{j}^{*}}\left|\delta \nabla u_{1}\right|^{2}\right)^{1 / 2}
\end{aligned}
$$

as in [FKP, p. 87], with $\varepsilon_{j}=\varepsilon\left(\ell\left(R_{j}\right)\right)$ replacing $\varepsilon_{0}$.

Now the key estimate $\left(f_{R_{j}^{*}}|F(y)|^{2} d y\right)^{1 / 2} \leq \varepsilon_{j} M_{\omega_{0}} S_{\beta} u_{1}\left(q_{0}\right)$ along with $|\nabla F| \lesssim\left|\nabla u_{1}\right|+\left|\nabla u_{0}\right|$ gives

$$
\begin{aligned}
\int_{R_{j}}|\nabla F(y)|^{2} \delta(y)^{2-n} d y \lesssim & \varepsilon_{j} M_{\omega_{0}} S_{\beta} u_{1}\left(q_{0}\right) \cdot\left(f_{R_{j}^{*}} \delta(y)^{2}|\nabla F(y)|^{2}\right)^{1 / 2} \\
& +\varepsilon_{j}^{2} M_{\omega_{0}} S_{\beta} u_{1}\left(q_{0}\right) \cdot\left(f_{R_{j}^{*}}\left|\delta \nabla u_{1}\right|^{2}\right)^{1 / 2} \\
& +\varepsilon_{j}\left(f_{R_{j}^{*}}\left|\delta \nabla u_{1}\right|^{2}\right)^{1 / 2}\left(f_{R_{j}^{*}}\left|\delta \nabla u_{0}\right|^{2}+f_{R_{j}^{*}}\left|\delta \nabla u_{1}\right|^{2}\right)^{1 / 2} .
\end{aligned}
$$

The terms involving $f_{R_{j}^{*}}\left|\delta \nabla u_{0}\right|^{2}$ can be handled by using

$$
\begin{aligned}
f_{R_{j}^{*}}\left|\delta \nabla u_{0}\right|^{2} & \lesssim \int_{R_{j}^{*}}\left|\nabla u_{0}(y)\right|^{2} \delta(y)^{2-n} \lesssim \int_{R_{j}^{*}} \frac{1}{\omega_{0}\left(\Delta_{j}^{*}\right)} \cdot \frac{\omega_{0}\left(\Delta_{j}^{*}\right)}{\delta(x)^{n-2}}\left|\nabla u_{0}(y)\right|^{2} \\
& \lesssim \frac{1}{\omega_{0}\left(\Delta_{j}^{*}\right)} \int_{R_{j}^{*}} G_{0}(0 ; y)\left|\nabla u_{0}(y)\right|^{2} d y \leq C\|f\|_{\mathrm{BMO}}
\end{aligned}
$$

according to Kenig's theorem, because $R_{j}^{*} \subseteq T_{r_{j}}\left(q_{0}\right)$ and $\omega_{0}$ is a doubling measure, so $\omega_{0}\left(\Delta_{r_{j}}\right) \sim \omega_{0}\left(\Delta_{j}^{*}\right)$, where $\Delta_{j}^{*}=\left.\operatorname{proj}\right|_{\partial B_{1}} R_{j}^{*}, r_{j}=\operatorname{diam} R_{j}$.

Thus $f_{R_{j}^{*}}\left|\delta \nabla u_{0}\right|^{2} \leq C=C\left(\|f\|_{\mathrm{BMO}}\right)$ and this gives

$$
\begin{aligned}
\int_{R_{j}}|\nabla F(y)|^{2} \delta(y)^{2-n} d y \lesssim \varepsilon_{j} M_{\omega_{0}} S_{\beta} u_{1}\left(q_{0}\right) & \\
& +\varepsilon_{j} M_{\omega_{0}} S_{\beta} u_{1}(q) \cdot\left(f_{R_{j}^{*}}\left|\delta \nabla u_{1}\right|^{2}\right)^{1 / 2}+\varepsilon_{j}\left(f_{R_{j}}\left|\delta \nabla u_{1}\right|^{2}\right)^{1 / 2} \\
& +\varepsilon_{j}^{2} M_{\omega_{0}} S_{\beta} u_{1}(q) \cdot\left(f_{R_{j}}\left|\delta \nabla u_{1}\right|^{2}\right)^{1 / 2}+\varepsilon_{j}\left(f_{R_{j}^{*}}\left|\delta \nabla u_{1}\right|^{2}\right) .
\end{aligned}
$$


Summing over $j$ with $R_{j} \cap \Gamma_{\alpha}\left(q_{0}\right) \neq \emptyset$ on both sides gives

$$
S_{\alpha} F\left(q_{0}\right)^{2} \lesssim C\left(M_{\omega_{0}} S_{\beta} u_{1}\left(q_{0}\right)+M_{\omega_{0}}^{2} S_{\beta} u_{1}\left(q_{0}\right)\right)
$$

using $\sum \varepsilon_{j} \leq \varepsilon_{0}$ and $\left(\sum \varepsilon_{j}^{2}\right)^{1 / 2} \leq \varepsilon_{0}$, Cauchy-Schwarz and the fact that $S_{\beta} u_{1}\left(q_{0}\right) \lesssim M_{\omega_{0}} S_{\beta} u_{1}\left(q_{0}\right)$. Taking square roots gives the required inequality.

To prove

$$
\left(f_{R_{j}}|F(y)|^{2} d y\right)^{1 / 2} \lesssim \varepsilon\left(\ell\left(R_{j}\right)\right) M_{\omega_{0}} S u_{1}\left(q_{0}\right), \quad q_{0} \in \operatorname{proj}\left(R_{j}\right),
$$

the stopping time argument that $[\mathrm{FKP}]$ use to prove that $\tilde{N}(F)(q) \leq$ $\varepsilon_{0} M_{\omega_{0}} S u_{1}(q)$ a.e. $d \sigma$ actually gives this estimate. The notation is that of $[\mathrm{FKP}]$.

Dividing the potential $F(x)=\int_{B_{1}(0)} \nabla_{y} G(x ; y) \cdot\left(\left[\varepsilon_{i j}(y)\right] \nabla u\right)$ into the same regions as in $[\mathrm{FKP}]$,

$$
\int_{B_{\delta(x) / 4}(x)}+\int_{\Omega_{0}}+\sum_{j=1}^{N} \int_{\Omega_{j} \cap \Gamma\left(q_{0}\right)}+\sum_{j=1}^{N} \int_{\Omega_{j} \cap \Gamma\left(q_{0}\right)^{\mathrm{c}}}+\int_{B_{1}(0) \backslash B_{1 / 2}\left(q_{0}\right)}
$$

one has only to notice that in

$$
\int_{B_{\delta(x) / 4}(x)} \nabla_{y} G(x ; y) \cdot\left[\varepsilon_{i j}(y)\right] \nabla u(y)
$$

the estimate on $\varepsilon(y)$ actually gives $\varepsilon(\delta(x))$ because $y \in B_{\delta(x) / 4}(x)$. So one has

$$
\left(f_{R_{j}}\left|F_{1}(x)\right|^{2} d x\right)^{1 / 2} \leq \varepsilon(\delta(x))\left(\int_{R_{j}^{*}}\left|\nabla u_{1}(y)\right|^{2} \delta(y)^{2-n}\right)^{1 / 2} .
$$
term

For $\int_{\Omega_{0}}$ the stopping time argument uses the Carleson condition on the

$$
\sum_{I \in I_{k}} \int_{I^{+}} \frac{a(y)^{2}}{\delta(y)^{2}} G(0 ; y) d y
$$

where $I$ is a "dyadic" cube in $\Delta_{0}, I_{k}$ is the collection of cubes in $\Delta_{0}$ with side length $\sim 2^{-k} \delta(x)$, and $I^{+}$is the upper half of the Carleson box associated with $I$. So

$$
\int_{I^{+}} \frac{a(y)^{2}}{\delta(y)^{2}} G(0 ; y) d y \leq \varepsilon(\ell(I)) \omega_{0}(I) \leq \varepsilon(\delta(x)) \omega_{0}(I)
$$

because of the definition of $\Omega_{0}=B_{\delta(x) / 2}\left(q_{0}\right) \cap B_{1}(0)$. So one has the estimate

$$
\left|\int_{\Omega_{0}} \nabla G(x ; y) \cdot\left[\varepsilon_{i j}\right] \nabla u(y)\right| \leq \varepsilon(\delta(x)) M_{\omega_{0}} S u_{1}\left(q_{0}\right) .
$$


For the integrals over $\Omega_{j} \cap \Gamma\left(q_{0}\right)$, condition (Cc) is used on

$$
\left(\int_{\Omega_{j} \cap \Gamma\left(q_{0}\right)} \frac{a(y)^{2}}{\delta(y)^{2}} G_{0}(0 ; y)\right)^{1 / 2} \leq \varepsilon\left(2^{j} \delta(x)\right) \omega_{0}\left(\Delta_{j}\right)^{1 / 2}
$$

to give

$$
\left|\int_{\Omega_{j} \cap \Gamma\left(q_{0}\right)} \nabla G_{0}(x, y)\left[\varepsilon_{i j}(y)\right] \nabla u_{1}(y) d y\right| \lesssim 2^{-j \alpha} \varepsilon\left(2^{j} \delta(x)\right) \cdot S u_{1}\left(q_{0}\right)
$$

with every other part of the argument the same as in $[\mathrm{FKP}]$. Now since $\varepsilon\left(2^{j} \delta(x)\right) \leq 2^{\gamma j}(\delta(x))$, we have

$$
\left|\int_{\Omega_{j} \cap \Gamma\left(q_{0}\right)} \nabla G_{0} \cdot\left[\varepsilon_{i j}\right] \nabla u_{1}\right| \leq 2^{-(\alpha-\gamma) j} \delta(x)^{\gamma} \cdot S u_{1}\left(q_{0}\right)
$$

and as $\gamma<\alpha$, one can sum over $j$ to get

$$
\left|\sum_{j=1}^{N} \int_{\Omega_{j} \cap \Gamma\left(q_{0}\right)} \nabla G_{0} \cdot\left[\varepsilon_{i j}\right] \nabla u_{1}\right| \lesssim \delta(x)^{\gamma} S u_{1}\left(q_{0}\right) .
$$

Similar estimates on the Green function $G_{0}(x ; y)$ along with the stopping time argument imply that

$$
\left|\sum_{j=1}^{N} \int_{\Omega_{j} \cap \Gamma\left(q_{0}\right)^{\mathrm{c}}} \nabla G_{0} \cdot\left[\varepsilon_{i j}\right] \nabla u_{1}\right| \lesssim \varepsilon(\delta(x)) M_{\omega_{0}} S u_{1}\left(q_{0}\right) .
$$

Finally, the fact that

$$
\left|\int_{B_{1}(0) \backslash B_{1 / 2}\left(q_{0}\right)} \nabla G_{0} \cdot\left[\varepsilon_{i j}\right] \nabla u_{1} d y\right| \leq C \int_{\partial B_{1} \backslash \Delta\left(q_{0}, 1 / 2\right)} S u_{1}(q) d \omega^{x}(q)
$$

shows that this part of the potential is bounded by $C \delta(x)^{\alpha} M_{\omega_{0}} S u_{1}\left(q_{0}\right)$ by using the estimate

$$
\frac{d \omega^{x}}{d \omega^{0}}(q) \lesssim \frac{2^{-\alpha j}}{\omega^{0}\left(\Delta_{j}\right)} \quad \text { if } q \in \Delta_{j} \backslash \Delta_{j-1}, \quad \Delta_{j}=\left\{q:\left|q-q_{0}\right| \lesssim 2^{j} \delta(x)\right\} .
$$

For $N$ such that $2^{N} \delta(x)=1 / 2$ we have

$$
\begin{aligned}
& \int_{\partial B_{1} \backslash \Delta\left(q_{0}, 1 / 2\right)} S u_{1}(q) d \omega^{x}(q)=\sum_{j=N-1}^{M} \int_{R_{j}} S u_{1}(q) d \omega^{x}(q) \\
& \leq \sum_{j=N}^{\infty} \frac{2^{-j \alpha}}{\omega^{0}\left(\Delta_{j}\right)} \int_{\Delta_{j}} S u_{1} d \omega^{0} \leq C \cdot 2^{-N \alpha} M_{\omega_{0}} S u_{1}\left(q_{0}\right) \lesssim \delta(x)^{\alpha} M_{\omega_{0}} S u_{1}\left(q_{0}\right) .
\end{aligned}
$$

This finishes the proof of the Main Lemma.

Finally, we prove a version of the good- $\lambda$ inequality (see [FKP, Lemma 2.16]) as a result of independent interest. 
Let

$$
\begin{aligned}
E=\left\{q \in \Delta_{r}: S_{\alpha} F(q)>\beta_{0} \lambda,\right. & F_{\beta^{\prime}}^{*}(q) M_{\omega_{0}} S_{\beta^{\prime}} u_{1}(q) \leq(\gamma \lambda)^{2}, \\
& \left.\widetilde{N}_{\beta^{\prime}}(\delta \nabla F)(q) \cdot M_{\omega_{0}} S_{\beta^{\prime}} u_{1}(q) \leq(\gamma \lambda)^{2}\right\} .
\end{aligned}
$$

Then for $\Delta_{r}$ a Whitney cube in the decomposition of $\left\{q: S_{\alpha} F(q)>\lambda\right\}$ one has

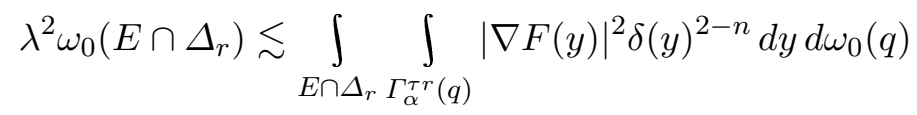

since $S_{\alpha} F(q)>\lambda \Rightarrow S_{\alpha}^{\tau r} F(q)>\lambda / 2$ on $E_{r}=E \cap \Delta_{r}$ for any $0<\tau<1$, $r=\delta(x)$, if $\gamma$ is chosen to be small enough (see [DJK]).

As in $[\mathrm{FKP}]$, with $A=\left[a_{i j}\right]$ the coefficient matrix for $L_{0}$,

$$
\begin{aligned}
\int_{E \cap \Delta_{r}}\left(S_{\alpha}^{\tau r} F(q)\right)^{2} d \omega_{0}(q) \lesssim \int_{\Omega}\left(\frac{1}{2} L_{0} F^{2}-F L_{0} F\right) G_{0}(0 ; y) d y \\
\lesssim \quad \int_{\partial \Omega} F(y) G_{0}(0 ; y) A \nabla F \cdot \vec{n}-\frac{1}{2} \int_{\Omega} A \nabla F^{2} \cdot \nabla G_{0} \\
\quad-\int_{\partial \Omega} F G_{0}\left[\varepsilon_{i j}\right] \nabla u_{1}(y) \cdot \vec{n}+\int_{\Omega} \nabla\left(F G_{0}\right) \cdot\left[\varepsilon_{i j}\right] \nabla u_{1}(y) \\
=\int_{\partial \Omega} F(y) G_{0}(0 ; y) A \nabla F(y) \cdot \vec{n}-\int_{\partial \Omega} \frac{1}{2} F^{2} A \nabla G_{0} \cdot \vec{n} \\
\quad+\int_{\Omega} \frac{1}{2} F(y)^{2} L_{0} G_{0}-\int_{\partial \Omega} F G_{0}\left[\varepsilon_{i j}\right] \nabla u_{1} \cdot \vec{n} \\
\quad+\int_{\Omega} \nabla F \cdot\left[\varepsilon_{i j}\right] \nabla u_{1}(y) G_{0}(y)+\int_{\Omega} F(y) \nabla G_{0}(y) \cdot\left[\varepsilon_{i j}\right] \nabla u_{1}(y) .
\end{aligned}
$$

Now

$$
\Omega=\bigcup_{q \in E \cap \Delta_{r}} \Gamma_{\alpha}^{\tau r}(q)
$$

so $0 \notin \Omega$ and $\int_{\Omega} F(y)^{2} L_{0} G_{0}=0$.

This leaves three boundary integrals and two integrals over $\Omega$ to be estimated. To handle the boundary integrals, which technically may not exist since $\nabla F$ and $\nabla G_{0}$ exist as $L_{\text {loc }}^{2}\left(B_{1}(0)\right)$ functions, one can use averaging over cones as in $[\mathrm{FKP}]$ : Take $\alpha<\beta<\beta^{\prime}$; then

$$
\begin{aligned}
\int_{\Delta_{r} \cap E}\left(S_{\alpha}^{\tau r} F(q)\right)^{2} d \omega_{0}(q) & =\int_{E_{r}} \int_{\Gamma_{\alpha}^{\tau r}(q)}|\nabla F(y)|^{2} \delta(y)^{2-n} d y d \omega_{0}(q) \\
& \lesssim \int_{E_{r}} \frac{1}{\beta-\alpha} \int_{\alpha}^{\beta} \int_{\Gamma_{\eta}^{\tau r}(q)}|\nabla F(y)|^{2} \delta(y)^{2-n} d y d \eta d \omega_{0}(q)
\end{aligned}
$$




$$
\begin{aligned}
& \simeq \frac{1}{\beta-\alpha} \int_{\alpha}^{\beta} \int_{E_{r}} \int_{\Gamma_{\eta}^{\tau r}(q)}|\nabla F(y)|^{2} \delta(y)^{2-n} d y d \omega_{0}(q) d \eta \\
& \lesssim \frac{1}{\beta-\alpha} \int_{\alpha}^{\beta} \int_{\Omega_{\eta}}(A \nabla F(y)) \cdot \nabla F(y) \delta(y)^{2-n} \omega_{0}\left(\Delta_{\delta(y)}\left(y^{*}\right)\right) d y d \eta
\end{aligned}
$$

where

$$
\Omega_{\eta}=\bigcup_{q \in E_{r}} \Gamma_{\eta}^{\tau r}(q), \quad E_{r}=\Delta_{r} \cap E .
$$

Now averaging over the top of $\Omega_{\eta}$ as well means that for $I(y) \geq 0$ each boundary integral can be replaced by

$$
\frac{1}{\beta-\alpha} \int_{\alpha}^{\beta} \int_{\partial \Omega_{\eta}} I(y) d y d \eta \lesssim \int_{N\left(\partial \Omega_{\eta}\right)} \frac{1}{\delta(y)} I(y) d y \lesssim \int_{\Omega_{\beta}} \frac{1}{\delta(I)} I(y) d y,
$$

where $N\left(\partial \Omega_{\eta}\right)$ is a solid neighborhood of $\partial \Omega_{\eta}^{\tau r} \subseteq \Omega_{\beta}^{\tau r}=\Omega_{\beta}$.

Obviously

$$
\frac{1}{\beta-\alpha} \int_{\alpha}^{\beta} \int_{\Omega_{\eta}} I(y) d y d \eta \leq \int_{\Omega_{\beta}} I(y) d y .
$$

So one needs to estimate the following five integrals:

$$
\begin{aligned}
& \int_{\Omega_{\beta}} \frac{1}{\delta(y)}|F(y)|\left|G_{0}(0 ; y)\right||\nabla F(y)|, \\
& \int_{\Omega_{\beta}} \frac{1}{\delta(y)}|F(y)|^{2}\left|\nabla G_{0}(0 ; y)\right|, \\
& \int_{\Omega_{\beta}} \frac{1}{\delta(y)}|F(y)|\left|G_{0}(0 ; y)\right|\left|\left[\varepsilon_{i j}\right] \nabla u_{1}(y)\right|, \\
& \int_{\Omega_{\beta}}\left|G_{0}(0 ; y)\right||\nabla F(y)| \varepsilon(y)\left|\nabla u_{1}(y)\right|, \\
& \int_{\Omega_{\beta}}|F(y)|\left|\nabla G_{0}(0 ; y)\right| \varepsilon(y)\left|\nabla u_{1}(y)\right| .
\end{aligned}
$$

For (1),

$$
\begin{aligned}
\int_{\Omega_{\beta}} & \frac{1}{\delta(y)}|F(y)|\left|G_{0}(0 ; y)\right||\nabla F(y)| d y=\sum_{I \in \mathcal{D}\left(\Delta_{r}\right)} \int_{I^{+} \cap \Omega_{\beta}} \frac{1}{\delta(y)}|F|\left|G_{0}\right||\nabla F| \\
& \lesssim \sum_{I \in \mathcal{D}\left(\Delta_{r}\right)} \frac{1}{\ell(I)} \frac{\omega_{0}(I)}{\ell(I)^{n-2}}\left(f_{I^{+} \cap \Omega_{\beta}}|F(y)|^{2} d y\right)^{1 / 2}\left(f_{I^{+} \cap \Omega_{\beta}}|\delta \nabla F|^{2}\right)^{1 / 2} \ell(I)^{n-1}
\end{aligned}
$$




$$
\begin{aligned}
& \lesssim \sum_{I \in \mathcal{D}\left(\Delta_{r}\right)} \frac{\ell(I)}{\ell(I)} \omega_{0}(I) \varepsilon(\ell(I)) \cdot M_{\omega_{0}} S_{\beta} u_{1}\left(q_{E \cap I}\right) \cdot \tilde{N}_{\beta}(\delta \nabla F)\left(q_{E \cap I}\right) \\
& \lesssim \gamma^{2} \lambda^{2} \sum_{k} \sum_{\substack{\ell(I)=2^{-k_{r}} \\
I \in \mathcal{D}\left(\Delta_{r}\right)}} \omega_{0}(I) \cdot \varepsilon(\ell(I)) \lesssim \gamma^{2} \lambda^{2} \omega_{0}\left(\Delta_{r}\right)
\end{aligned}
$$

since diam $\Omega_{\beta} \sim r$ and by hypothesis

$$
\sum_{\substack{k=0 \\ \ell(I)=2^{-k} r}}^{\infty} \varepsilon(\ell(I)) \leq C_{0} .
$$

Here $q_{E \cap I}$ can be any point in $E \cap I$ for $\beta^{\prime}>\beta$ sufficiently large, and $\mathcal{D}\left(\Delta_{r}\right)$ is the collection of all dyadic subcubes of $\Delta_{r}$.

The integral (2) can also be estimated:

$$
\begin{aligned}
\int_{\Omega_{\beta}} \frac{1}{\delta(y)} F(y)^{2} \mid & \nabla G_{0}(0 ; y)\left|d y=\sum_{I \in \mathcal{D}\left(\Delta_{r}\right)} \int_{I^{+} \cap \Omega_{\beta}} \frac{1}{\delta} F^{2}\right| \nabla G_{0} \mid \\
& \lesssim \sum_{I \in \mathcal{D}\left(\Delta_{r}\right)} \frac{1}{\ell(I)} F^{*}\left(q_{E \cap I}\right) \frac{\omega_{0}(I)}{\ell(I)^{n-1}}\left(f_{I^{+} \cap \Omega_{\beta}}|F|^{2}\right)^{1 / 2} \cdot \ell(I)^{\frac{n}{2} \cdot 2} \\
& \lesssim \sum_{I \subseteq \mathcal{D}\left(\Delta_{r}\right)} \omega_{0}(I) F_{\beta^{\prime}}^{*}\left(q_{E \cap I}\right) \varepsilon(\ell(I)) M_{\omega_{0}} S_{\beta^{\prime}}\left(q_{E \cap I}\right) \\
& \lesssim \gamma^{2} \lambda^{2} \sum_{j} \sum_{\ell(I)=2^{-j} r} \varepsilon(\ell(I)) \omega_{0}(I)=C \gamma^{2} \lambda^{2} \omega_{0}\left(\Delta_{r}\right)
\end{aligned}
$$

as above.

For (3),

$$
\begin{aligned}
\int_{\Omega_{\beta}} & \frac{1}{\delta(y)}|F(y)|\left|G_{0}(0 ; y)\right||\varepsilon(y)|\left|\nabla u_{1}(y)\right| d y \\
& =\sum_{I \in \mathcal{D}\left(\Delta_{r}\right)} \int_{I^{+} \cap \Omega_{\beta}} \frac{1}{\delta(y)}|F|\left|G_{0}\right||\varepsilon|\left|\nabla u_{1}\right| \\
& \lesssim \sum_{I \in \mathcal{D}\left(\Delta_{r}\right)} \varepsilon(\ell(I)) \frac{\omega_{0}(I)}{\ell(I)^{n-1}}\left(\int_{I^{+} \cap \Omega_{\beta}}|F(y)|^{2} d y\right)^{1 / 2}\left(\int_{I^{+} \cap \Omega_{\beta}}\left|\nabla u_{1}(y)\right|^{2}\right)^{1 / 2} \\
& \lesssim \sum_{k} \sum_{\substack{\ell(I)=2^{-k} r \\
I \cap \Omega_{\beta} \neq \emptyset}} \varepsilon\left(2^{-k} r\right) \omega_{0}(I) \gamma^{2} \lambda^{2} \leq C \gamma^{2} \lambda^{2} \omega_{0}\left(\Delta_{r}\right) .
\end{aligned}
$$


For (4),

$$
\begin{aligned}
\int_{\Omega_{\beta}}\left|G_{0}(0 ; y)\right| & |\nabla F(y)| \varepsilon(y)\left|\nabla u_{1}(y)\right| \\
\leq & \sum_{I \in \mathcal{D}\left(\Delta_{r}\right)}\left(f_{I^{+} \cap \Omega_{\beta}}|\delta \nabla F(y)|^{2}\right)^{1 / 2}\left(f_{I^{+} \cap \Omega_{\beta}}\left|\delta \nabla u_{1}(y)\right|^{2}\right)^{1 / 2} \\
& \cdot \varepsilon(\ell(I)) \sup _{y \in I^{+}}\left|G_{0}(0 ; y)\right| \cdot \ell(I)^{n-2} \\
\lesssim & \sum_{I \in \mathcal{D}\left(\Delta_{r}\right)} \gamma^{2} \lambda^{2} \varepsilon(\ell(I)) \omega_{0}(I) \leq C \gamma^{2} \lambda^{2} \omega_{0}\left(\Delta_{r}\right) \quad \text { as before. }
\end{aligned}
$$

Finally, we estimate the fifth integral:

$$
\begin{aligned}
& \int_{\Omega_{\beta}}|F(y)|\left|\nabla G_{0}(0 ; y)\right| \varepsilon(y)\left|\nabla u_{1}(y)\right| \\
& =\sum_{I \in \mathcal{D}\left(\Delta_{r}\right)} \int_{I^{+} \cap \Omega_{\beta}}|F(y)|\left|\nabla G_{0}(0 ; y)\right||\varepsilon(y)|\left|\nabla u_{1}(y)\right| \\
& \lesssim \sum_{I \in \mathcal{D}\left(\Delta_{r}\right)} F_{\beta^{\prime}}^{*}\left(q_{E \cap I}\right) \cdot \varepsilon(\ell(I))\left(f_{I^{+} \cap \Omega_{\beta}}\left|\delta \nabla G_{0}\right|^{2}\right)^{1 / 2}\left(f_{I^{+} \cap \Omega_{\beta}}\left|\delta \nabla u_{1}\right|^{2}\right)^{1 / 2} \ell(I)^{n-2} \\
& \lesssim \sum_{I \in \mathcal{D}\left(\Delta_{r}\right)} F_{\beta^{\prime}}^{*}\left(q_{E \cap I}\right) \cdot S_{\beta^{\prime}} u_{1}\left(q_{E \cap I}\right) \cdot \varepsilon(\ell(I)) \sup _{y \in I^{+}}\left|G_{0}(0 ; y)\right| \ell(I)^{n-2} \\
& \lesssim \gamma^{2} \lambda^{2} \sum_{j} \sum_{\ell(I)=2^{-j} r} \varepsilon(\ell(I)) \omega_{0}(I) \lesssim C \gamma^{2} \lambda^{2} \omega_{0}\left(\Delta_{r}\right) .
\end{aligned}
$$

So $\lambda^{2} \omega_{0}\left(E_{r}\right) \leq C \gamma^{2} \lambda^{2} \omega_{0}\left(\Delta_{r}\right)$. Dividing by $\lambda^{2}$ and summing over the Whitney cubes $\Delta_{r}$ gives $\omega_{0}(E) \leq C \gamma^{2} \omega_{0}(S F>\lambda)$.

This is enough to prove

$$
\begin{aligned}
\int_{\partial B_{1}}(S F(q))^{p} d \omega_{0}(q) \lesssim & \int_{\partial B_{1}}(\tilde{N}(F)(q))^{p} d \omega_{0}(q) \\
& +\int_{\partial B_{1}}(\tilde{N}(\delta \nabla F)(q))^{p} d \omega_{0}(q)+\int_{\partial B_{1}} f(q)^{p} d \omega_{0}(q)
\end{aligned}
$$

using the maximal theorem, the estimates

$$
F^{*}(q) \leq \widetilde{N}(F)(q)+u_{0}^{*}(q), \quad S_{\beta} u_{1}(q) \leq S_{\beta} F(q)+S_{\beta} u_{0}(q)
$$

and standard arguments.

It can be noted here that the same kind of argument proves the good- $\lambda$ inequality for parabolic functions also given the proper decay of the Carleson coefficient. 
Appendix. We recall that the spherical cone is

$$
\Gamma_{\gamma}^{\delta}\left(\theta_{x}\right)=\left\{(r, \theta): \delta<r<1,\left|\theta-\theta_{x}\right|<\gamma(1-r)\right\},
$$

and the Euclidean cone is

$$
\begin{aligned}
& \Gamma_{\gamma}^{\delta}(x)=\left\{y:\left|y-r^{*} \theta_{x}\right|<\gamma\left(1-r^{*}\right)\right. \text { where } \\
& \left.1-r^{*}=\operatorname{dist}\left(y, \mathbb{R}^{n-1}\right) \text { and } \operatorname{dist}\left(y, \text { center of } S^{n-1}\right) \geq \delta\right\} .
\end{aligned}
$$

Here $\mathbb{R}^{n-1}$ is tangent to $S^{n-1}$ at $\theta_{x}=x$.

We will show that for $\gamma$ fixed there are apertures $\beta, \beta^{\prime}$ so that $\Gamma_{\beta^{\prime}}^{\delta}\left(\theta_{x}\right) \subset$ $\Gamma_{\gamma}^{\delta}(x)$ and $\Gamma_{\gamma}^{\delta}(x) \subset \Gamma_{\beta}^{\delta}\left(\theta_{x}\right)$.

Claim. $\left|r-r^{*}\right|<1-r \leq 1-r^{*}$ for $y=r \theta$.

It is enough to show this in $\mathbb{R}^{2}$. Take $\theta_{x}=x=0$ and $S^{1}$ centered at $(0,1)$.

Let $(x, y)=\vec{y}$ lie on a circle of radius $r=\varrho$ about $(0,1)$, where $\varrho$ is large enough to have this circle intersect $\Gamma_{\alpha}^{\delta}(0)$. The line $y=\alpha x$ intersects the circle $x^{2}+(y-1)^{2}=\varrho^{2}$ at $x$ such that $x^{2}+(\alpha x-1)^{2}=\varrho^{2}$. Solving gives

$$
x=\frac{2 \alpha \pm \sqrt{4 \alpha^{2}-4\left(\alpha^{2}+1\right)\left(1-\varrho^{2}\right)}}{2\left(\alpha^{2}+1\right)} .
$$

Choosing the point on the circle nearest 0 gives

$$
\vec{y}=\left(\frac{\alpha-\sqrt{-1+\varrho^{2}\left(\alpha^{2}+1\right)}}{\left(\alpha^{2}+1\right)}, \frac{\alpha^{2}-\alpha \sqrt{-1+\varrho^{2}\left(\alpha^{2}+1\right)}}{\left(\alpha^{2}+1\right)}\right)=\left(x, 1-r^{*}\right)
$$

so

$$
\begin{aligned}
\left|r-r^{*}\right| & =\mid \varrho-\left(1-\frac{\left.\alpha^{2}-\alpha \sqrt{-1+p^{2}\left(\alpha^{2}+1\right)}\right)}{\left(\alpha^{2}+1\right)}\right), \\
\frac{\left|r-r^{*}\right|}{1-r} & =\left|\frac{\left(\alpha^{2}+1\right) \varrho+\alpha^{2}-\alpha \sqrt{-1+\varrho^{2}\left(\alpha^{2}+1\right)}-\left(\alpha^{2}+1\right)}{\left(\alpha^{2}+1\right)(1-\varrho)}\right| \\
& =\left|\frac{(\varrho-1)\left(\alpha^{2}+1\right)+\alpha^{2}-\alpha \sqrt{\varrho^{2}\left(\alpha^{2}+1\right)-1}}{\left(\alpha^{2}+1\right)(1-\varrho)}\right| .
\end{aligned}
$$

Using l'Hospital we obtain

$$
\lim _{\varrho \uparrow 1} \frac{\left|r-r^{*}\right|}{1-r}=\lim _{\varrho \rightarrow 1}\left|\frac{\left(\alpha^{2}+1\right)-\frac{\alpha \varrho\left(\alpha^{2}+1\right)}{\sqrt{\varrho^{2}\left(\alpha^{2}+1\right)-1}}}{\alpha^{2}+1}\right|=1-\frac{\alpha}{\sqrt{\alpha^{2}+1-1}}=0 .
$$

So there is a height $\delta_{0}$ so that $\left|r^{*}-r\right|<1-r$ if $r \geq \delta_{0}$.

The fact that $1-r \leq 1-r^{*}$ is obvious so if $y \in \Gamma_{\gamma}^{\delta}\left(\theta_{x}\right)$ then $\left|\theta_{y}-\theta_{x}\right|<$ $\gamma(1-r)$, hence $\left|r \theta_{y}-r^{*} \theta_{x}\right|<r \gamma(1-r)+\left|r^{*} \theta_{x}-r \theta_{x}\right|=r \gamma(1-r)+\left|r^{*}-r\right|\left|\theta_{x}\right|$ and so $\left|y-r^{*} \theta_{x}\right|<\left(\gamma+\left|\theta_{x}\right|\right)\left(1-r^{*}\right)$, that is, $y \in \Gamma_{\beta^{\prime}}^{\delta}(x)$ if $\beta^{\prime} \geq 2 \gamma+\left|\theta_{x}\right|$. 
Conversely, if $y \in \Gamma_{\beta}^{\delta}(x)$ then $\left|y-r^{*} \theta_{x}\right|<\gamma\left(1-r^{*}\right)$ and

$$
\left|r \theta_{y}-r \theta_{x}\right| \leq \gamma\left(1-r^{*}\right)+\left|r \theta_{x}-r^{*} \theta_{x}\right|
$$

so that

$$
\left|\theta_{y}-\theta_{x}\right| \leq \frac{\gamma}{r}(1-r)+\frac{\gamma}{r}\left(r-r^{*}\right)+\frac{\left|\theta_{x}\right|}{r}\left|r-r^{*}\right|
$$

and hence

if $\beta \geq\left(2 \gamma+\left|\theta_{x}\right|\right) / \delta_{0}$.

$$
\left|\theta_{y}-\theta_{x}\right|<\frac{2 \gamma}{\delta_{0}}(2(1-r))+\frac{\left|\theta_{x}\right|}{\delta_{0}}(1-r) \leq \beta(1-r)
$$

\section{References}

[BM] R. Bañuelos and C. Moore, Probabilistic Behavior of Harmonic Functions, Birkhäuser, 1999.

[CWW] S.-Y. A. Chang, J. M. Wilson and T. Wolff, Some weighted norm inequalities concerning the Schrödinger operator, Comment. Math. Helv. 60 (1985), $217-246$.

[DJK] B. Dahlberg, D. Jerison and C. Kenig, Area integral estimates for elliptic differential operators with nonsmooth coefficients, Ark. Mat. 22 (1984), 97-108.

[FKP] R. Fefferman, C. Kenig and J. Pipher, The theory of weights and the Dirichlet problem for elliptic equations, Ann. of Math. 134 (1991), 65-124.

[H] L. L. Helms, Introduction to Potential Theory, Wiley, 1969.

[K] C. Kenig, Harmonic Analysis Techniques for Second Order Elliptic Boundary Value Problems, CBMS Reg. Conf. Ser. Math. 83, Amer. Math. Soc., 1994.

[S] E. Stein, Singular Integrals and Differentiability Properties of Functions, Princeton Univ. Prdss, 1970.

[CS1] C. Sweezy, L-harmonic functions and the exponential square class, Pacific J. Math. 147 (1991), 187-200.

[CS2] - $B^{q}$ for parabolic measures, Studia Math. 131 (1998), 115-135.

Department of Mathematical Sciences

New Mexico State University

Las Cruces, NM 88003, U.S.A.

E-mail: csweezy@nmsu.edu 